\title{
Le cerf et le roi : modèle sacrificiel et rite d'intronisation dans l'ancien Mexique
}

\section{Guilhem Olivier}

\section{Q OpenEdition}

\section{Journals}

Édition électronique

URL : https://journals.openedition.org/jsa/9573

DOI : $10.4000 /$ jsa.9573

ISSN : 1957-7842

Éditeur

Société des américanistes

\section{Édition imprimée}

Date de publication : 15 juillet 2008

Pagination : 191-230

ISSN : 0037-9174

\section{Référence électronique}

Guilhem Olivier, "Le cerf et le roi : modèle sacrificiel et rite d'intronisation dans l'ancien Mexique », Journal de la Société des américanistes [En ligne], 94-1 | 2008, mis en ligne le 10 juin 2013, consulté le 03 septembre 2022. URL : http://journals.openedition.org/jsa/9573; DOl : https://doi.org/10.4000/ jsa.9573 


\title{
LE CERF ET LE ROI : MODÈLE SACRIFICIEL ET RITE D'INTRONISATION DANS L'ANCIEN MEXIQUE
}

\author{
Guilhem OLIVIER *
}

Après une brève description des rites préliminaires à la chasse et des techniques cynégétiques, cet article prétend établir un parallèle entre les cerfs chassés et les guerriers capturés et sacrifiés. De même, certains aspects des rites d'intronisation suggèrent que le futur roi était assimilé à une victime sacrificielle suivant un modèle qui pourrait renvoyer à celui du cerf immolé, peut-être en tant qu'ancêtre. [Mots-clés : Mésoamérique, cerf, chasse, sacrifice, intronisation.]

El venado y el rey : modelo sacrificial y rito de entronización en el México antiguo. Después de una breve descripción de los ritos previos a la cacería y de las técnicas cinegéticas, este artículo pretende establecer un paralelo entre los venados cazados y los guerreros capturados y sacrificados. Así mismo, algunos aspectos de los ritos de entronización sugieren que el futuro rey era concebido como una víctima sacrificial, según un modelo que podría remitir al del venado inmolado, tal vez en su aspecto de antepasado. [Palabras claves : Mesoamérica, venado, cacería, sacrificio, entronización.]

The deer and the king : sacrificial model and enthronment rite in Ancient Mexico. After a brief description of the rituals previous to hunting and the cynegetic techniques, this article pretends to establish a parallel between hunted deers and captured and sacrified warriors. As well, some aspects of the enthronment rites suggest that the future king was assimilated to a sacrified victim, according to a model who could refer to an immolated deer, may be in its ancestor aspect. [Key words: Mesoamerica, deer, hunting, sacrifice, enthronment.]

Un rituel singulier et touchant, réalisé par les Quiché de Mazatenango au début du XvIII ${ }^{\mathrm{e}}$ siècle, a été décrit par Fray Francisco Vásquez (1938, II, pp. 9495) dans sa Crónica de la provincia del Santísimo Nombre de Jesús de Guatemala... :

* Instituto de Investigaciones Históricas, Ciudad de Investigación en Humanidades, 3er circuito cultural universitario, Ciudad universitaria, 04510 México DF [olivier@servidor.unam.mx].

Journal de la Société des Américanistes, 2008, 94-1, pp. 191-230. OS Société des Américanistes. 
[...] Llegó [el fraile] al paraje que buscaba, que era la espaciosa placeta que hacía un frondoso árbol. Al pie de éste estaba un altar formado del altor de los nuestros; pero de carrizos y ramas, y de él salia un respaldo a modo de sitial todo enrejado de hojas y flores. En el altar estaba, en uno como baúl fabricado de cañas, y cundido de flores, un venado, que aquel dia habian cogido, que era a quien adoraban y hacian aquel culto, ofreciéndole perfumes y todo el pueblo velándole, sentadas las indias en el suelo con sus niños y niñas, y los indios como en corro, haciendo danzas, al son de aquellas tristes flautas.

Au-delà de ce témoignage d'un culte rendu à un cerf, un tant soit peu idyllique quoique sans doute fidèle, la figure du cervidé me semble idéale à bien des égards pour étudier les rapports étroits et complexes qui existaient entre la chasse, la guerre et le sacrifice en Mésoamérique ${ }^{1}$. Malgré l'abondance des sources, la littérature scientifique, mis à part quelques articles ou extraits d'ouvrages, ne comporte pas, à ma connaissance, d'études complètes sur le cerf ou la chasse dans l'ancien Mexique. Il n'est donc pas inutile de présenter, dans un premier temps, quelques données sur la chasse au cervidé, notamment quant à ses aspects rituels. On insistera sur le traitement rituel des crânes de cerfs qui se révèle proche de celui assigné aux crânes des ennemis sacrifiés. Ensuite, il sera question des liens entre la chasse et la guerre et de l'équivalence, attestée dans de nombreux documents, entre le sacrifice du cerf et le sacrifice humain. La dernière partie sera consacrée à certains aspects des rituels d'accès au pouvoir et aux liens éventuels entre le sacrifice symbolique du futur gouvernant et celui du cervidé en tant qu'ancêtre.

\section{LA CHASSE AU CERF}

\section{Les rites préalables et le statut du chasseur}

Avant d'effectuer une expédition cynégétique et pour s'assurer de son succès, divers procédés divinatoires ont été et sont parfois encore utilisés en Mésoamérique. Les sources anciennes mentionnent la consultation de manuscrits pictographiques dans l'aire maya et le recours à des spécialistes du calendrier divinatoire, par exemple chez les anciens Zapotèques ou encore, au $\mathrm{xx}^{\mathrm{c}}$ siècle, chez les Mam et les Quiché du Guatemala, afin de déterminer les jours opportuns à l'obtention du gibier ${ }^{2}$. Par ailleurs, nous avons des témoignages anciens et modernes de l'emploi divinatoire de grains de maïs, de bâtonnets, de cordes à nœuds, de cristaux, de bézoards, voire de poils, du crâne et des bois du cerf, éléments révélateurs du moment et du lieu propices à la chasse ${ }^{3}$. Les signes scrupuleusement perçus indiquent, à l'occasion, les dons à adresser aux Maîtres des animaux ou aux divinités de la chasse pour s'assurer leur concours, offrandes quantifiées dans une section du Codex Cospi (1994, pl. 21-31) ou encore dans un livret que conservaient les Chontal dans les années $1950^{4}$. N'oublions pas les rêves prémonitoires des chasseurs, notamment ceux à caractère érotique où la femme possédée annonce la proie offerte, ainsi que les signes observés en chemin 
qui revêtent une extrême importance pour entreprendre ou retarder une entreprise cynégétique ${ }^{5}$.

Des invocations anciennes prononcées avant de chasser le cerf ont été conservées dans l'œuvre de Ruiz de Alarcón (1987) et dans El Ritual de los Bacabes (1987), les ethnologues ayant recueilli au $x^{\mathrm{e}}$ siècle des textes semblables chez les Lacandons, les Mayas de Chan Kom, les Chontal, les Huichol, etc. (Tozzer 1982 ; Redfield et Villa Rojas 1934 ; Carrasco 1960 ; Gutiérrez del Ángel 2002). De nombreuses entités divines sont sollicitées dans ces invocations, divinités protectrices des animaux et des montagnes qui souvent se confondent, dieu du feu, maîtres des instruments et armes utilisées pour la chasse (arc, flèche, lacet, etc.). Diverses offrandes sont réalisées à leur attention, sang provenant d'autosacrifice, fumée de copal, flèches votives, aliments variés, parfois de l'herbe soigneusement sélectionnée pour satisfaire le goût des cervidés.

Une documentation abondante se réfère à l'indispensable abstinence sexuelle des chasseurs. Des récits mazatèques, chinantèques, tlapanèques, mixe, nahua, et de divers groupes mayas expliquent les conséquences funestes des manquements à la morale sexuelle de la part des chasseurs, mais aussi de leurs épouses. Cas extrême, les Nahua de la région du Golfe du Mexique étendent ces prohibitions sexuelles à leur chien de chasse ! (Vázquez García et Godínez Guevara 2005). Attardons-nous sur ces interdits que James George Frazer (1981, I, pp. 593-614), il y a un siècle déjà, avait longuement décrits parmi divers peuples de chasseurs et de pêcheurs. Le savant écossais (ibid., p. 597) signalait fort justement que « [...] on paraît supposer en général que le mauvais effet de l'incontinence n'est pas d'affaiblir celui qui s'y livre, mais d'offenser, pour une raison ou pour une autre, les animaux qui, en conséquence, ne se laisseraient pas prendre ». En effet, ces rituels préalables à la chasse et les récits qui sanctionnent les transgressions sexuelles s'inscrivent dans un modèle général d'échange de type matrimonial entre le Maître des animaux et le chasseur. Les connotations érotiques de la chasse sont particulièrement évidentes en Mésoamérique. En témoigne, entre autres exemples, l'invocation maya conservée dans El Ritual de los Bacabes (1987, pp. 419-420) où le chasseur anticipe, dans un langage particulièrement explicite, un acte sexuel avec une biche. Les déesses de l'amour, patronnes des prostituées, Tlazolteotl et Xochiquetzal, n'apparaissent-elles pas pourvues de bois de cerf dans l'iconographie ancienne? (Codex Borgia 1963, pl. 59 ; Codex Laud 1966, pl. 42) (Figure 1). Reprenant les excellentes analyses de Braakhuis (2001), on peut considérer que la proie est assimilée à l'épouse du chasseur, le Maître des animaux se transformant en beau-père que le chasseur doit rétribuer au moyen d'offrandes et par une conduite morale irréprochable ${ }^{6}$. Dans un mythe pipil recueilli par Leonhard Schultze-Jena (1977, pp. 30-31), le «service » dû au Maître des animaux consiste en la régénération des os des cerfs morts, le chasseur épousant la fille-biche du Maître et le couple prolifique « [...] teniendo cada día dos venaditos $[\ldots] » !^{7}$ 


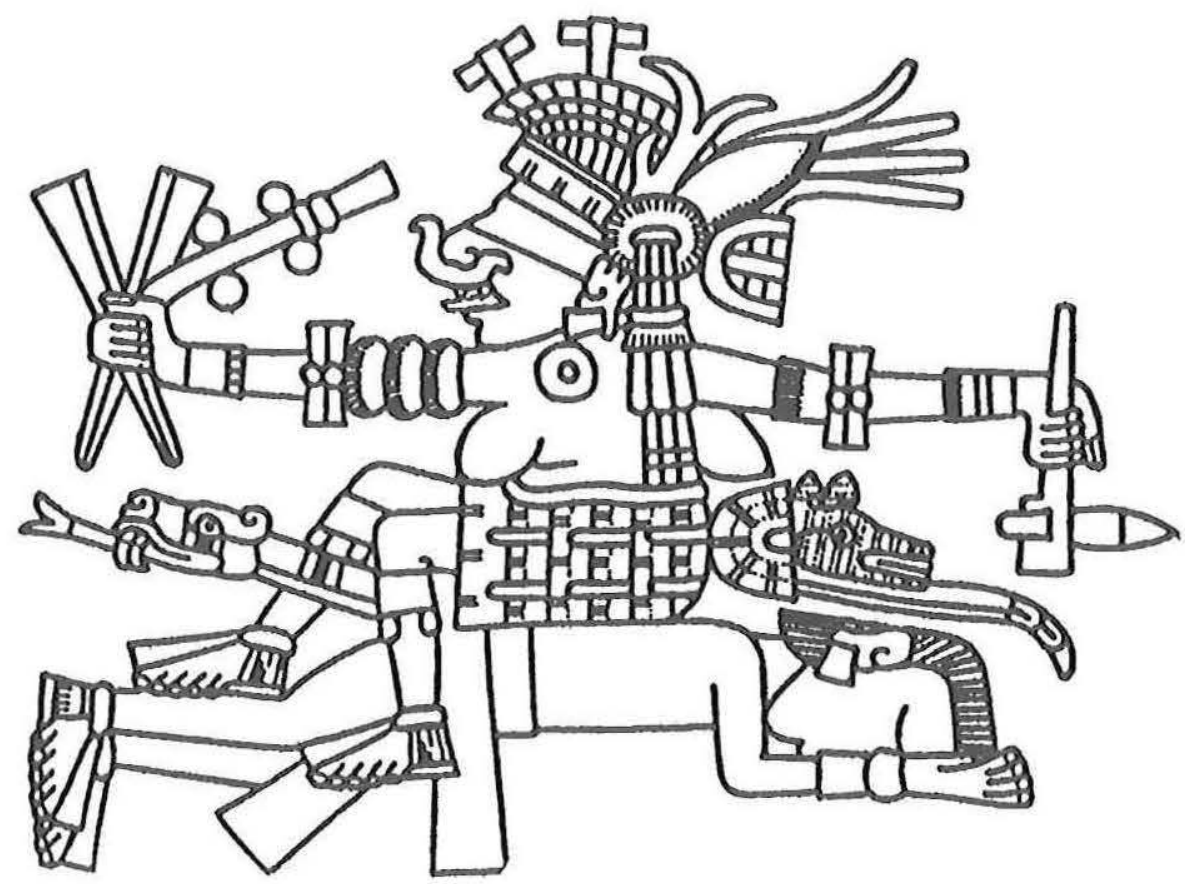

Fig. 1 - Tlazolteotl, déesse de l'amour charnel, portant des bois de cerf (d'après Codex Laud 1966, pl. 42).

\section{Les techniques de chasse}

Elles étaient variées, comme l'exprime ce passage des informateurs de fray Bernardino de Sahagún (CF, X, p. 179) relatif aux Otomi : «ils vont tuant les cerfs avec des flèches, les capturant avec des filets, les capturant avec des pièges [...] (maçamjntinemj, maçamatlavitinemj, tlatlâpeoalhujtinemj $[\ldots]$ ) ${ }^{8}$. Extrêmement répandu en Mésoamérique à partir du Postclassique, l'usage d'arcs et de flèches est illustré dans les Primeros Memoriales (Sahagún 1993, fol. 252r), où des chasseurs ornés comme des Mimixcoa sont représentés avec ces armes, alors qu'ils participent à la chasse collective organisée durant la vingtaine de quecholli (Figure 2). Autre arme de jet plus ancienne, le propulseur est parfois employé pour chasser le cerf dans l'aire maya (Codex Madrid 1977, pl. 38-41). Ce manuscrit pictographique illustre également l'emploi de lacets pour capturer les cervidés (ibid., pl. 42-49), une pratique attestée pour l'ensemble de la Mésoamérique selon divers vocabulaires anciens et de nombreux témoignages ethnographiques, incluant à l'occasion d'intéressants mythes d'origine de cette technique 


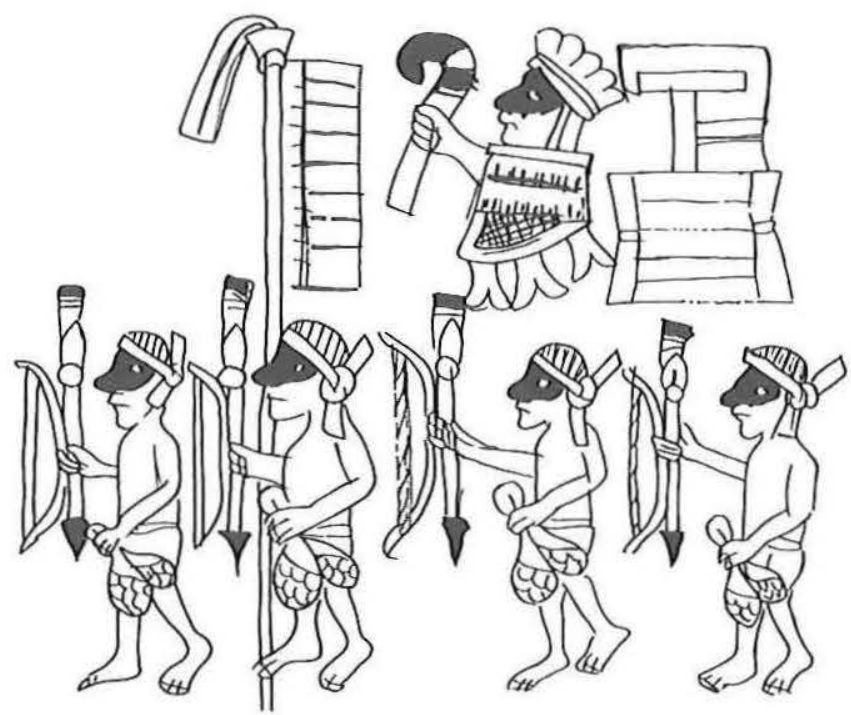

FIG. 2 - Chasseurs ornés comme des Mimixcoa durant la vingtaine de quecholli (d'après Sahagún, Primeros Memoriales 1997, fol. 252r).

(Figure 3) ${ }^{9}$. Il est question également de pièges, cavités pourvues ou non de pieux, lesquels peuvent être dissimulés sur les bords de chemins étroits où l'on contraint les cerfs à passer. Certains chasseurs revêtaient la peau d'un cerf afin de s'approcher de leurs congénères et ainsi de les tuer plus aisément ${ }^{10}$. Signalons au passage l'existence de sifflets spéciaux destinés à attirer les cervidés, appeaux dont le symbolisme sexuel renvoie à l'importante dimension érotique de la chasse que j'ai signalée plus haut (Olivier 1999-2000, pp. 85-86). Enfin, les sources révèlent la participation de chiens dressés pour la chasse au cerf, notamment en pays maya (Landa 1959, p. 135 ; López de Cogolludo 1954, I, p. 339 ; Ruz 1996, p. 107 ; Vázquez García et Godínez Guevara 2005).

L'ample diffusion en Mésoamérique de chasses collectives a retenu l'attention des chroniqueurs. Ainsi, dans la région de Justlahuaca (Oaxaca),

[...] ninguno podía ir a caza, sino cuando el señor iba. Y dicen que cuando así había de salir a caza el señor, antes que saliese de su casa, hacía la junta de su gente e imocaban a sus idolos. Y luego salian al monte y cazaban dos o tres dias, y mataban muchos venados, conejos, leones, tigres, gallinas de la tierra monteses, y otras muchas sabandijas. (Relación de Justlahuaca in Relaciones geográficas del siglo xv: Antequera 1984, p. 288)

Fray Toribio Benavente ou Motolinía (1971, p. 281) décrivit avec admiration les chasseurs purépecha qui acculaient les animaux dans un espace restreint avant de les tuer. Dans le nord-est de l'actuel Mexique, les Indiens brûlaient la végéta- 


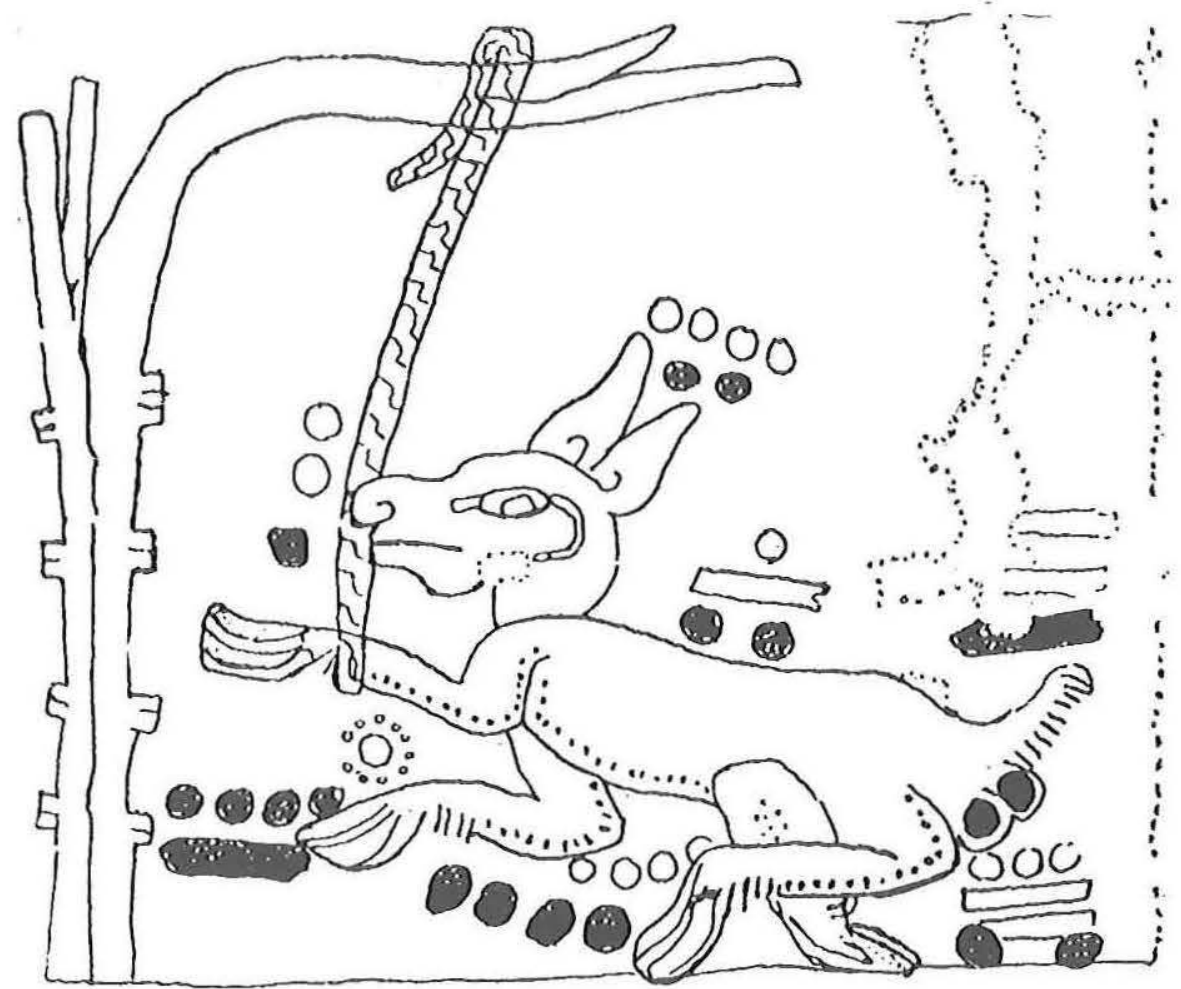

Fig. 3 - Utilisation de lacets pour capturer les cerfs (d'après Codex Madrid 1977, pl, 46).

tion pour contraindre leurs futures proies à se regrouper (Fernández de Oviedo 1945, X, pp. 221-222). On organisait également des chasses collectives dans la vallée de Mexico: par exemple, Motecuhzoma II - par ailleurs chasseur confirmé, fort habile à la sarbacane disait-on - fut autorisé, durant sa captivité, à poursuivre ses activités cynégétiques :

[...] cuando iba a la caza de montería le llevaban en hombros con las guardas de españoles y tres mil indios tlaxcaltecas; acompañábanle los señores sus vasallos, por hacerle solaz ; banqueteaba a éstos y a los españoles con mucha gracia, dando a los unos y a los otros muchos dones y haciéndoles muchas mercedes. (Cervantes de Salazar 1985, p. 334)

De cette façon, le souverain mexica ne faisait que prolonger artificiellement son rôle de guide rituel des chasseurs durant certaines fêtes religieuses. Au cours de la vingtaine de quecholli, déjà mentionnée, une battue était organisée sur la montagne de Zacatepec à laquelle le roi participait, revêtu des insignes du dieu de 
la chasse Mixcoatl (Figure 2). Après le sacrifice des animaux capturés, le tlatoani récompensait les meilleurs chasseurs, prodigalité que Motecuhzoma II réitéra à l'issue de la chasse collective réalisée en compagnie de ses vainqueurs (CF, II, pp. 136-140; Codex Tudela 1980, fol. $24 \mathrm{r}^{\circ}$ ).

L'espace manque pour décrire les rituels suscités par la réception des cerfs chassés, généralement accueillis par des prières où l'on remercie le Maître des animaux ${ }^{11}$ ainsi que le cervidé qui s'est " offert » aux chasseurs ${ }^{12}$; des excuses sont murmurées, on caresse tendrement l'animal, parfois orné de fleurs, de pierres précieuses, revêtu d'habits ou protégé avec des couvertures; encensé avec du copal, le cerf est l'objet d'offrandes de boissons et d'aliments, le cas échéant placés délicatement dans sa bouche ${ }^{13}$. Le retour d'expéditions cynégétiques peut aussi donner lieu à des rituels collectifs, ainsi les Mayas yucatèques

[...] invocaban a los dioses de la caza Acanum, Zuhuyzib Zip y Tabay y otros, y repartíanles el incienso, el cual echaban al brasero; y en tanto que ardia, sacaba cada uno una flecha y una calavera de venado, las cuales untaban los chaces con el betín azul. Ya untadas bailaban con ellas en las manos; otros se horadaban las orejas, otros la lengua [...]. Habiendo hecho esto primero, el sacerdote y los oficiales de la fiesta ofrecian luego los dones, y así bailando se escanciaba el vino y' emborrachaban hechos unos cestos. (Landa 1959, pp. 92-94)

Il conviendrait d'entreprendre une étude détaillée du traitement sacrificiel et culinaire du cerf. Je citerai simplement une excellente description incluse dans un rapport du Licenciado Palacio à propos du Guatemala :

Lo que hacian en los sacrificios de la pesca y caza era que tomaban un venado vivo y llevábanlo al patio del cu e iglesia que tenian fuera del pueblo, y lo ahogaban y lo desollaban y le sacaban toda la sangre en una olla, y el higado y bofes y buches lo hacian pedazos muy menudos, el corazón, cabeza y pies apartaban. Y mandaban cocer el venado por sí y la sangre por si y, mientras esto se cocía, hacían su baile. Tomaban el papa y sabio la cabeza del venado por las orejas, y los cuatro pies, y el mayordomo en un brasero llevaba el corazón (se quemaba con hule y copal), e incensaba al idolo que tenía puesto y señalado para la caza y pesca. Acabado el mitote, ofrecían la cabeza y pies al idolo y chamuscábanla. Y después de chamuscada, la llevaban a casa del papa y se la comía. Y el venado y sangre comian delante del idolo, u otro cualquier animal que sacrificase [...]. (in Relaciones geográficas del siglo xvı : Guatemala 1982, pp. 280-281)

Cette "cuisine du sacrifice » du cerf - pour reprendre le titre du célèbre ouvrage de Jean-Pierre Vernant et Marcel Detienne (1979) - mériterait une analyse fine réalisée avec d'autres matériaux comparatifs, notamment ethnographiques, les données précises de ce type étant hélas rares dans la littérature ancienne. 
DESTIN ET « RECYCLAGE 》 DES CRÂNES ET DES OS DE CERFS... ET DE GUERRIERS

\section{Les rituels mortuaires des cerfs}

Revenons sur l'utilisation rituelle des crânes de cerfs. Au retour de la battue effectuée en quecholli, les chasseurs mexica les suspendaient aux poutres de leurs maisons (CF, II, p. 137). À près de 500 ans de distance, les Mixe de Oaxaca et les Chorti du Honduras procèdent de la même façon avec les crânes des animaux qu'ils ont tués (Miller 1956, pp. 253-254; Wisdom 1961, p. 97). Quant aux Tolupan du Honduras, ils attachent les crânes et les os longs de leurs proies à un pilier de leur maison (Chapman 1978, pp. 132-133). Walter S. Miller (ibid.) précise que les chasseurs mixe, une fois par an, déposent ces crânes dans la montagne, pratique attestée aujourd'hui encore chez les Triqui de Oaxaca ${ }^{14}$. À la fin du XIX ${ }^{\mathrm{e}}$ siècle, les Zapotèques de Caxonos (Oaxaca) et les Mixe de San Juan Guichocovi (Oaxaca) plaçaient les têtes des cerfs tués sur des pierres spéciales situées dans les montagnes (Gillow 1889, p. 212 ; Starr 1900, p. 55). En revanche, les Huichol et les Mexicaneros à la même époque disposaient les crânes des cervidés dans des grottes (Lumholtz 1986, I, p. 507 ; II, p. 172 ; Preuss 1998, p. 206) et ainsi font encore les Tlapanèques en ce début du $\mathrm{XxI}^{\mathrm{e}}$ siècle (Dehouve 2006a, p. 201).

Une variante intéressante consiste à traiter rituellement non pas les crânes de cerfs mais leurs mâchoires. Au début du $x^{\mathrm{e}}$ siècle, Alfred M. Tozzer (1982, p. 134) observa dans les maisons des Lacandons, " [...] pegadas al techo varias quijadas de animales, especialmente de venados, monos y jabalies, las cuales sin duda sirven de recordatorios, o posiblemente como cuentas de los sacrificios de carne que se han hecho a los dioses ${ }^{15}$. Après avoir tué sept cerfs, les chasseurs nahua d'Ichcatepec (Veracruz) avaient l'obligation de remettre à la Terre les mâchoires inférieures de ces cervidés qu'ils déposaient au sommet d'une montagne, accompagnées d'offrandes (Reyes García 1960, p. 37). Bien qu'il conviendrait d'examiner les rapports des archéologues de manière systématique, une mâchoire de cerf utilisée comme collier a été exhumée dans la tombe VIII-36 de Copan (Fash 1991, pp. 92-93).

\section{Une comparaison avec les rites mortuaires des guerriers}

Plusieurs indices suggèrent que le traitement rituel des crânes de cervidés était équivalent à celui réalisé avec les crânes d'ennemis sacrifiés. De même que les Mayas yucatèques dansaient avec leurs trophées de chasse, les Mexica brandissaient les têtes des guerriers ou représentants de dieux sacrifiés durant des danses religieuses (CF, II, pp. 54, 156 ; Durán 1995, II, p. 85). Quant au fémur d'un captif exceptionnel, suspendu à une poutre, il faisait l'objet d'un culte sous le nom 
de Malteotl, « Dieu captif » (CF, II, p. 60 ; Durán 1995, I, p. 217). Les chroniques mentionnent également la pratique qui consistait à ficher les crânes des sacrifiés dans les murs ou les toits des temples ${ }^{16}$. On songe aux Cora qui, avant la conquête, "[...] reservando sólo las cabezas de los más abultados [des cerfs chassés], para colgarlas en los Templos de los Idolos » (Ortega 1996, p. 20). Guerriers exceptionnels, les Cora dansaient eux aussi avec les têtes tranchées de leurs ennemis qu'ils conservaient ensuite chez eux. Dans le même contexte, Arias de Saavedra (1990, p. 295), à qui nous devons ces données, ajoute que l'on perçait la cloison nasale du valeureux qui avait capturé un prisonnier ("le agujereaban las narices »), lui attribuant le titre prestigieux de « Capitán ». Nous reviendrons plus loin sur cette importante cérémonie. Un traitement rituel différent était réservé par les anciens Nicarao aux crânes de leurs victimes sacrificielles, ils les plaçaient " [...] en unos palos que están fronteros de los adoratorios é templos» (Fernández de Oviedo 1945, XI, p. 93). À la lecture de ce procédé, la tentation est grande d'évoquer la coutume de placer les têtes ou les crânes des guerriers sacrifiés sur des structures en bois, les célèbres tzompantli mexica, que l'on a fort justement comparés à des arbres (Graulich 2005, pp. 265-267, 321). De fait, au XVII ${ }^{\mathrm{e}}$ siècle, les Acaxee de Durango suspendaient " algún hueso » de leurs ennemis sur un sapotillier (Santarén in González 1980, p. 375), tandis que leurs voisins, les Xixime, avaient coutume « [...] con los huesos y calaveras [des Acaxee !] celebrar sus triunfos, y colgarlos a las paredes, y puertas de sus casas, y de los árboles que tenían vecinos a ellas » (Pérez de Ribas 1992, p. 531). Nous retrouvons donc, dans ce témoignage exceptionnel, les différents lieux designés comme espaces privilégiés où étaient placés les crânes et les os, aussi bien des cerfs chassés que des prisonniers de guerre sacrifiés.

L'utilisation de mâchoires de prisonniers de guerre est moins connue. Toutefois, fray Diego de Landa (1959, p. 52) signale que les Mayas yucatèques " [...] después de la victoria quitaban a los muertos la quijada y limpia de la carne, poníansela en el brazo ». Durant les fouilles du temple de Quetzalcoatl à Teotihuacan ont été découverts des colliers de mâchoires humaines, associés à des personnages sacrifiés, ainsi que d'autres colliers de mâchoires réalisées en coquillage (Sugiyama 1991, pp. 307, 318) ${ }^{17}$.

Au-delà de l'utilisation comme " trophées » des crânes des animaux chassés, les rites durant lesquels ils étaient manipulés ainsi que les lieux où ils étaient déposés révèlent l'intention de susciter la renaissance des animaux chassés (de même que des ennemis rituellement exécutés !). Il s'agissait sans doute de provoquer le retour à la vie des proies en remettant au Seigneur de la montagne et des animaux les crânes et/ou les os de ses créatures (Olivier 1997, pp. 52-53; Dehouve 2006a, p. 201) ${ }^{18}$. Comme l'écrit Braakhuis (2001, p. 395), " [...] hunt can be viewed as a cycle in which flesh is reduced to bone and bone is regenerated as flesh $》$. De même, Françoise Neff Nuixa (2001, pp. 367-368) décrit comment les Tlapanèques du Guerrero couvrent les os des animaux chassés avec du coton pour les 
« habiller » ${ }^{19}$. Akuun Júba, le Maître des animaux, recevait dans l'anfractuosité d'une montagne les crânes de ces proies arrosés de sang de dindon, afin d'assurer ensuite leur renaissance. Le symbolisme des montagnes et des grottes, où sont déposés les crânes et les os de cerfs, est bien connu, elles sont la demeure du Maître des animaux et matrice où se régénère la vie (Heyden 1991) ${ }^{20}$. D'une certaine façon, on peut considérer que ces « restes solides » des proies sont semés avant leur renaissance, les os étant assimilés à des semences (Graulich 1987, p. 113) ${ }^{21}$.

Comment expliquer dès lors l'usage de placer les crânes et les mâchoires, non pas dans les grottes ou sur les montagnes, mais suspendus aux poutres des maisons? Il a été question des têtes ou crânes des guerriers disposés sur des piliers, des arbres ou encore des tzompantli assimilés aussi à des arbres. De ce fait, les crânes pourraient représenter les fruits-semences d'où les guerriers vont renaître, un peu à l'image du crâne du dieu quiché Hun Hunahpu qui, placé dans un arbre, féconda Ixquic, future mère des jumeaux divins (Popol Vuh 1985, p. 114). Il n'est pas interdit de penser que les poteaux et les poutres des maisons partageaient cette même symbolique. De fait, au $\mathrm{XvI}^{\mathrm{e}}$ siècle, les habitants de Teotitlan (Oaxaca) célébraient de cette façon la fête de titil :

[...] salian a caza con sus arcos y flechas; y toda la caza que mataban de venados y conejos, y culebras y ratones y otras aves, lo colgaban en un árbol grande y, de alli lo tomaban entre todos y lo traían en peso hasta ponerlo delante del idolo: iban, delante, tañendo y cantando, y, llegados al idolo, hincaban en el suelo el árbol y lo quemaban con toda la caza que en él habia. (Relación geográfica de Teutitlan in Relaciones geográficas del siglo XVI : Antequera, 1984b, p. 201)

Or nous savons que la crémation dans l'ancien Mexique était liée à l'idée de renaissance, comme l'atteste par exemple le célèbre mythe d'origine du Soleil et de la Lune. Quant aux Lacandons, ils racontent comment, jadis, les chasseurs déposaient les os de leurs proies au pied d'un arbre, à partir desquels le dieu Hach Ak Yum ressuscitait les animaux (Boremanse 1986, p. 102). Les Navajo font de même avec les bois du premier cerf tué, les crânes étant placés sur les branches d'un sapin ou d'un acajou. L'explication d'un chasseur navajo se passe de commentaires : " [...] as soon as the humters leave, these heads will turn into deer again. These deers will go back to their home " (Hill 1938, pp. 113, 117, 121) ${ }^{22}$. Nous pouvons ajouter au dossier un beau mythe des Tzutuhil du Guatemala : à l'origine il y avait un grand arbre situé au centre du monde qui portait toutes choses vouées à l'existence telles que rochers, fruits, maïs, éclairs, fragments de temps ainsi que des cerfs suspendus à ses branches (Carlsen et Prechtel 1991, p. 27). Comment ne pas songer au concept de Tlalocan-Tamoanchan, source de vie où sont « recyclés» les morts et les futures semences des plantes et des animaux ? (López Austin 1994). Or ces espaces mythiques peuvent apparaître sous la forme d'une grotte (Tlalocan) ou encore d'un arbre (Tamoanchan). Enfin, élément fondamental qui nous renvoie à l'équivalence entre les ennemis captifs et 
les cerfs chassés ${ }^{23}$, les mythes mexica affirment que les Mimixcoa, prototypes des guerriers morts à la guerre ou sacrifiés, assimilés à des cervidés, naquirent... précisément au Tlalocan ! ${ }^{24}$

\section{L'ÉQUIVALENCE DU SACRIFICE DU CERF ET DU SACRIFICE HUMAIN}

Pour mieux comprendre cette équivalence, il faut rappeler que la chasse et la guerre étaient étroitement imbriquées, ces deux activités étant destinées à obtenir des proies-captifs pour être sacrifiés (Graulich 1997) ${ }^{25}$. Les mythes rendent compte de cette assimilation, ainsi dans le récit d'origine de la " Guerre sacrée ». Les 400 Mimixcoa pourvus d'armes magnifiques par le Soleil n'offrirent pas leur gibier à Soleil et Terre, en outre ils couchèrent avec des femmes et s'enivrèrent. Soleil créa alors cinq autres Mimixcoa, armés de façon rustique, qui chassèrent leurs frères aînés comme s'ils étaient du gibier. Ils furent immolés à Soleil et Terre, selon un modèle que nous retrouvons dans de nombreux rituels sacrificiels (Leyenda de los Soles 1992a, p. 92 ; 1992b, pp. 150-151 ; Historia de los mexicanos por sus pinturas 1941, pp. 216-217).

Plusieurs témoignages iconographiques et écrits présentent des cerfs sacrifiés par arrachement du cœur, ce qui pourrait constituer un nouvel indice en faveur d'un traitement sacrificiel identique à celui réservé aux guerriers captifs. Signalons le cervidé sacrifié entre deux coyotes sur une peinture de Techinantitla (Teotihuacan), ainsi qu'une représentation du Codex Nuttall (1992, pl. 44), où le célèbre souverain mixtèque " 8 Cerf » Griffe de Jaguar assiste au sacrifice par cardiectomie d'un coyote et d'un cerf. Détail significatif, la divinité solaire " 13 Roseau » est la destinataire du sacrifice, tout comme lors des sacrifices humains. Parmi les sources écrites, les minutes des procès inquisitoriaux de Mani (Yucatan) nous apprennent que dans le village d'Usil " [...]confesó el dicho cacique de su pueblo, que se llama Pedro Yah, que tenía gran temor porque habría dos años que él y otros cinco habían sacrificado un venado y sacándole el corazón y ofrecidosele a los ídolos » (Scholes et Adams 1938, vol. 1, p. 163). Dernier exemple, vers 1700, les Zapotèques de Caxonos furent surpris au milieu d'un rituel : "En el suelo habia una cierva grande tendida boca arriba y con la barriga destripada, que todavia se estaba meneando [...] " (Gillow 1889, p. 106). Selon Danièle Dehouve (2006b, p. 68), il s'agissait d'une cérémonie liée à l'accession au pouvoir d'un dirigeant indigène, l'immolation d'une biche n'étant que le substitut d'un sacrifice humain.

Dans un autre registre, l'équivalence entre les cerfs et les hommes sacrifiés pourrait rendre compte d'un type de menace que les Tlaxcaltèques adressèrent aux Tenochca durant le siège de Mexico-Tenochtitlán : "Ah perros, pués queréis morir como venados [...] » (Cervantes de Salazar 1985, p. 746), annonçant sans équivoque que les Tenochca allaient être sacrifiés. Deux siècles plus tard, en 1720, les guerriers cora prédisaient un même destin sacrificiel aux envahisseurs espagnols : face à eux, un Indien 
[... ] sin parar en sus movimientos, en sus amenazas, y amágos : ya se levantaba en el aire, ya se tiraba hasta la tierra, sin cesar de hablar, y alentar a los suyos, asegurandoles el vencimiento con decirles, que ya los Venados estaban cogidos en el cerco, y que antes de declinar el Sol, no había de quedar Español vivo. (Ortega 1996, p. 129)

La fête de quecholli, dont il a été déjà question, illustre de façon manifeste cette assimilation réciproque des prisonniers de guerre et des cervidés chassés. Le traitement des captifs sur le point d'être exécutés est éloquent à cet égard, les informateurs de Sahagún (1997, p. 64) à Tepepulco affirmant que « [...] ils attachaient des pieds et des mains le captif ou l'esclave baigné [...] (Auh in malli anoço tlaaltili quinmailpiaya : ioan quimicxilpiaya [...]) ». Incontestablement ils étaient liés à la façon des prises de chasse. En outre, dans le Codex de Florence, il est précisé que de ces captifs « [...] on dit, pour cela, qu'ils sont conquis, détruits comme des cerfs, ils imitent les cerfs qui ainsi meurent [...] (qujlmach ic qujnmaçapoloa, qujntlaehecalhuia in mamaça, in juh mjquj [...]) » (CF, II, p. 139). En revanche, d'après la description de fray Diego Durán (1995, II, p. 84), les animaux capturés durant la chasse collective étaient sacrifiés devant une statue de Mixcoatl « [...] al mesmo modo que de los hombres $[\ldots] \rrbracket^{26}$.

D'importantes données linguistiques révèlent des concepts similaires quant aux relations entre prises de chasse et captifs de guerre ${ }^{27}$. Les vocabulaires maya sont les plus explicites : à partir de l'analyse des termes associés à la chasse dans le remarquable dictionnaire cakchiquel de fray Thomás de Coto (1983), Mario Humberto Ruz (1996, p. 104) considère que « [...] la acción de cazar; qui canon, qui cako se liga con los activos tin canoh y tin cak, que en sentido estricto significan buscar, tirar; pero también con cana que designa la presa que se coge en la guerra, pues marca la acción de cautivar; de alli que se llamase cana tanto a presa como a cautivo [...] ". Les données yucatèques sont semblables, comme le manifestent les diverses significations du verbe chuc: « tuer à coup de flèches, chasser avec des pièges ou avec des chiens », mais aussi « capturer des ennemis » (Nagy 1997 , p. 58) ${ }^{28}$.

Identifiés aux victimes de sacrifice, les cerfs sont semblables aux Mimixcoa, premières victimes de la Guerre sacrée, prototypes des sacrifiés dans les codex et les sources écrites (Codex Borgia 1963, pl. 19, 21 ; Codex Boturini 1964, pl. 4 ; Chimalpain 1997, pp. 12-13 ; etc.) (Figure 4). Mixcoatl, dieu de la chasse, ne se présente-t-il pas dans les mythes comme une divinité vaincue? (Historia de los mexicanos por sus pinturas 1941, p. 217 ; Leyenda de los Soles 1992a, p. 94 ; 1992b, pp. 153-154). Dans une version purépecha, notre héros appelé ici Cupanzueri fut sacrifié suite à sa défaite au jeu de balle face aux seigneurs de l'inframonde. Son fils le ressuscita mais, effrayé par des cailles, Cupanzueri se transforma en cerf (Relación de Michoacán 1988, pp. 293-294; Graulich 1987, pp. 183-184). La diffusion de ce mythe chez les Indiens actuels est remarquable. Les Totonaques de la Sierra de Puebla racontent comment un fils retrouva la tombe de son père et le 

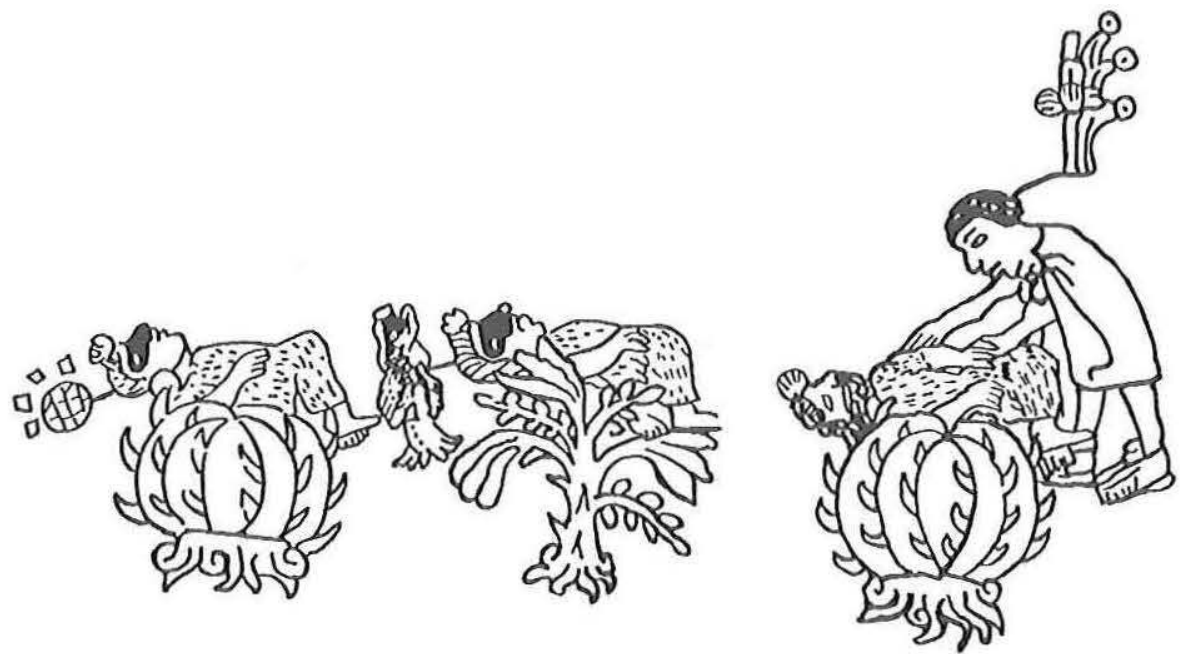

FIG, 4 - Sacrifice des Mimixcoa par les Mexicas (d'après Codex Boturini 1964, pl. 4).

ressuscita. Il le porta sur ses épaules mais la chute d'une feuille l'effraya et le père fut transformé en cervidé. Sur ces entrefaites, l'enfant lui cria : «Va donc, les hommes te tueront comme avant " (Ichon 1969, p. 66). Dans une version tepehua, le fils s'adresse ainsi à son père identiquement métamorphosé : « [...] pero ahora ni modo, los perros son para tí, las balas son para tí y las moscas también » (Williams García 1972, p. 91). Le destin du cerf est donc clairement assigné : la mort comme proie des chasseurs.

L'utilisation de nerfs de cerf pour confectionner des flèches renvoie à des conceptions similaires. Un passage de l'œuvre de Diego Muñoz Camargo (1998, pp. 98-99) raconte la formation miraculeuse de flèches dans le temple de Mixcoatl-Camaxtli, traits qui ensuite s'animèrent et attaquèrent seuls les ennemis des Tlaxcaltèques. Parmi les matériaux utilisés pour créer ces flèches singulières, on mentionne " [...] cañas de carrizo, y las arponadas lengïentas, varas tostadas, puntas, nervios de venado [... ] ». L'emploi de nerfs de cerfs est bien attesté dans les régions septentrionales du Mexique comme la Nueva Galicia et le Nuevo León ${ }^{29}$. Aujourd'hui encore les Mexicaneros de Durango utilisent des nerfs de cerf pour attacher les deux parties qui composent leurs flèches, l'une en roseau et l'autre en bois (Alvarado Solís 2004, pp. 52, 112). Or, parmi les « atrocités » attribuées aux Chichimèques, les chroniqueurs espagnols s'offusquent du fait que « [...] quitánles [a los cautivos] ansi mismo los nervios para con ello atar los pedernales en sus flechas » (Relación geográfica de Querétaro in Relaciones geográficas del siglo XVI: Michoacán 1987, p. 225). Au-delà de l'utilisation pratique de nerfs humains pour attacher les parties constitutives des flèches, il semble significatif de retrouver l'équivalence cerf/victime humaine à travers ces «techniques». Poussons plus 
avant notre raisonnement, les Mexicaneros assimilent les nerfs des cerfs qu'ils utilisent pour leurs flèches à des veines par où circule le sang (Alvarado Solís 2004, p. 247). Si ces flèches sont pourvues de "sang de cerf », l'utilisation de nerfs humains nous renvoie à l'identification par les anciens Nahua des flèches à des guerriers sacrifiés ou morts à la guerre (CF, VI, p. 224 ; Olivier 2004). En effet, les combattants tombés sur des champs de bataille lointains étaient incinérés sur place, et on effectuait avec leurs flèches, identifiées donc à ces vaillants défunts, les funérailles dans leur lieu d'origine (Motolinía [ou Benavente] 1971, p. 307$)^{30}$.

Une prohibition alimentaire significative va nous permettre d'approfondir le concept de l'identification du cerf à une victime humaine sacrifiée. D'après Alonso de León (1961, p. 21) qui, vers 1649, décrivit les mœurs des Indiens du Nuevo León, " [...] la carne del venado es la mejor que tienen ; y en matándolo, lo dejan y envían a sus mujeres al otro día, por él; y ellas por el rastro lo hallan y lo traen. Es suyo del cazador el cuero y no come la carne; repártense entre todos ». Le chasseur ne consomme donc pas la chair de sa proie. La même coutume a été décrite par Miguel del Barco (1988, p. 217) au sujet des Indiens de Basse Californie : "Cuando iban a caza de venados, y mataban alguno, el hombre que lo mató no comía de la carne de aquel venado, porque, si comía de ella, decian, no mataría en adelante más venados ». Cette pratique se retrouve chez les Chumash de Californie au XVIII ${ }^{\mathrm{e}}$ siècle ${ }^{31}$ ou encore chez les Mixe de Oaxaca décrits par Frederick Starr $\left(1900\right.$, p. 55) à la fin du XIX ${ }^{\mathrm{e}}$ siècle ${ }^{32}$. Il s'agit en fait d'un trait caractéristique de nombreuses sociétés de chasseurs, en Sibérie par exemple où Roberte Hamayon (200lb, p. 139) signale que le fait de consommer sa propre proie est assimilé à un inceste. Plusieurs spécialistes des sociétés amazoniennes ont relevé le même type de prohibition, le chasseur s'abstenant de manger la chair des animaux qu'il a lui-même tués (Lizot 1976, pp. 191, 208 ; Descola 1986, p. 321 ; Viveiros de Castro 1993, p. 413 ; Surrallés 2003, p. 131). Or ce système d'interdits s'étendait aux guerriers ayant fait des captifs, tels les Tupinamba au $\mathrm{Xvl}^{\mathrm{c}}$ siècle qui les excluaient du banquet cannibale (Viveiros de Castro 1993, pp. 413, 423).

Il est temps de revenir au Mexique central avec un témoignage exceptionnel en langue nahuatl des informateurs indigènes de Sahagún (CF, II, p. 54) relatif à la vingtaine de tlacaxipehualiztli :

Et celui qui possède un captif ne mangeait pas la chair de son captif. Il disait : vais-je donc me manger moi-même ? quand il capturait un captif, il disait : il est mon cher fils et le captif disait : il est mon cher père (Auh in male, amo uel qujquaia, yn jnacaio imal, qujtoaia, cujx çan no ne njnoquaz : ca yn iquac caci, qujtoa, ca iuhquj nopiltzin : Auh in malli, qujtoa ca notatzin).

Ce texte fondamental manifeste l'identité entre le chasseur-guerrier et sa proie-victime. Il confirme en outre qu'à travers une victime substitut, le sacrifiant offrait sa propre vie en sacrifice (Graulich 2005, pp. 165, 349; Olivier 2008, 
pp. 275-276). Enfin, les données concernant les prohibitions alimentaires affectant à la fois les cerfs et les guerriers capturés démontrent à nouveau l'équivalence entre le cerf et la victime humaine de sacrifice.

\section{LE GOUVERNANT SACRIFIÉ}

Nous ne disposons pas de l'espace nécessaire pour décrire et interpréter de manière détaillée les rites complexes d'intronisation du nouveau roi (tlatoani) mexica (Townsend 1987 ; Graulich 1994 ; Olivier 1997, 2008). Il convient toutefois de reprendre certains aspects de ces rites qui sont en rapport avec les concepts mésoaméricains de chasse, de guerre et de sacrifice. En résumant outrageusement des recherches antérieures, nous avons proposé qu'au cours des cérémonies d'intronisation le futur roi mexica réalisait au moins trois rituels durant lesquels il mourait symboliquement : réclusion et descente dans l'inframonde où il s'identifiait aux paquets sacrés de ses divinités tutélaires, Huitzilopochtli et Tezcatlipoca ; percement de la cloison nasale qui transformait le nouveau souverain en victime potentielle de sacrifice à l'image des Mimixcoa et enfin sacrifice de son premier captif auquel il s'identifiait et qui représentait une divinité solaire et ignée. Sacrifié en puissance, le roi apparaissait donc à l'issue des rites d'intronisation comme investi de qualités divines parmi lesquelles le destin sacrificiel était central (Olivier 2008).

Revenons sur le percement de la cloison nasale du candidat, sans doute l'un des moments clefs des cérémonies d'intronisation. Dans le cas de Motecuhzoma II, Durán (1995, I, pp. 459-460) décrit comment « [...] le sentaron en el trono real y supremo [...] y, horadándole las ternillas de las narices, le atravesaron en ellas una piedra delgada, a manera de canutillo, que por lo menos debia ser alguna esmeralda ». Dans les sources, plusieurs représentations et récits mythiques nous fournissent des éléments susceptibles d'éclaircir la signification de ce rituel. Ainsi, Karl Anton Nowotny (2005, pp. 274-275) a proposé qu'une planche du Codex Borgia (1963, pl. 44) pourrait être en rapport avec la cérémonie de percement de la cloison nasale : on y observe en effet un aigle, un jaguar et un quetzal - trois animaux associés symboliquement aux gouvernants - qui mordent le nez de trois personnages dont les visages émergent de la gueule d'un serpent (Figure 5). Le savant autrichien rapproche cette scène d'un passage de l'Historia toltecachichimeca (1976, pp. 160-172) où les Chichimèques furent intronisés comme tlatoque par les dirigeants toltèques Icxicoatl et Quetzaltehueyac. Dans un premier temps, les Chichimèques renversés sur des arbres (mezquites) furent alimentés par un aigle et un jaguar, prototypes des guerriers, mais aussi animaux symbolisant les principaux destinataires du sacrifice, Soleil et Terre. Plus tard, les chefs toltèques percèrent la cloison nasale des futurs dirigeants chichimèques (Figure 6). Enfin, un épisode de la migration mexica durant lequel les adorateurs 


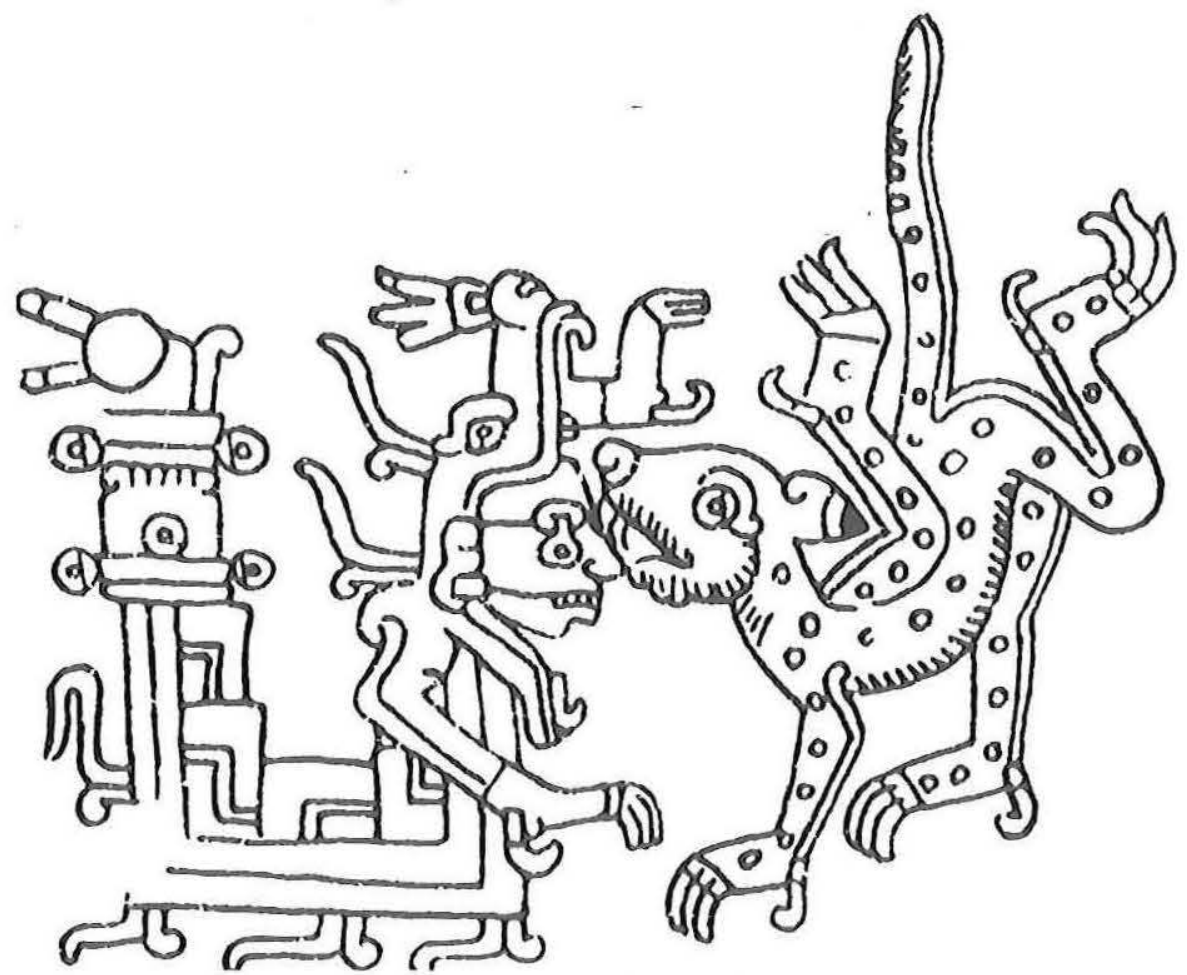

FIG. 5 - Un jaguar mord le nez d'un personnage dont le visage émerge d'une gueule de serpent (d'après Codex Borgia 1963, pl. 44).

de Huitzilopochtli sacrifièrent les Mimixcoa complète notre corpus. Signalons que ces personnages mythologiques sont renversés sur des cactus et des épineux, à l'instar des Chichimèques dans l'Historia tolteca-chichimeca (1976). Certes, le rituel du percement de la cloison nasale est absent dans cet épisode. Cela étant, d'autres événements autorisent un rapprochement avec les rites d'intronisation. D'une part, suite au sacrifice des Mimixcoa, les Aztèques adoptèrent le nom de "Mexica », d'autre part, ils reçurent des armes comme l'arc et la flèche, butin provenant de leurs victimes (Codex Boturini 1964, pl. 4 ; Codex Aubin 1963, pp. 21-23 ; Chimalpain 1997, pp. 14-15) (Figure 4). Rappelons que cet épisode de la migration mexica est calqué sur le sacrifice des Mimixcoa pour nourrir Soleil et Terre dans le mythe d'origine de la Guerre sacrée déjà mentionné. Quant au lien avec les rites d'intronisation, il est illustré par le fait que le roi adoptait un nouveau nom et recevait des armes lors de son intronisation, " [...] en señal de que prometía la defensa de su ciudad y el morir por ella »(Durán 1995, I, p. 113) ${ }^{33}$. Le rite qui consistait à percer la cloison nasale du futur dirigeant en vint à symboliser 


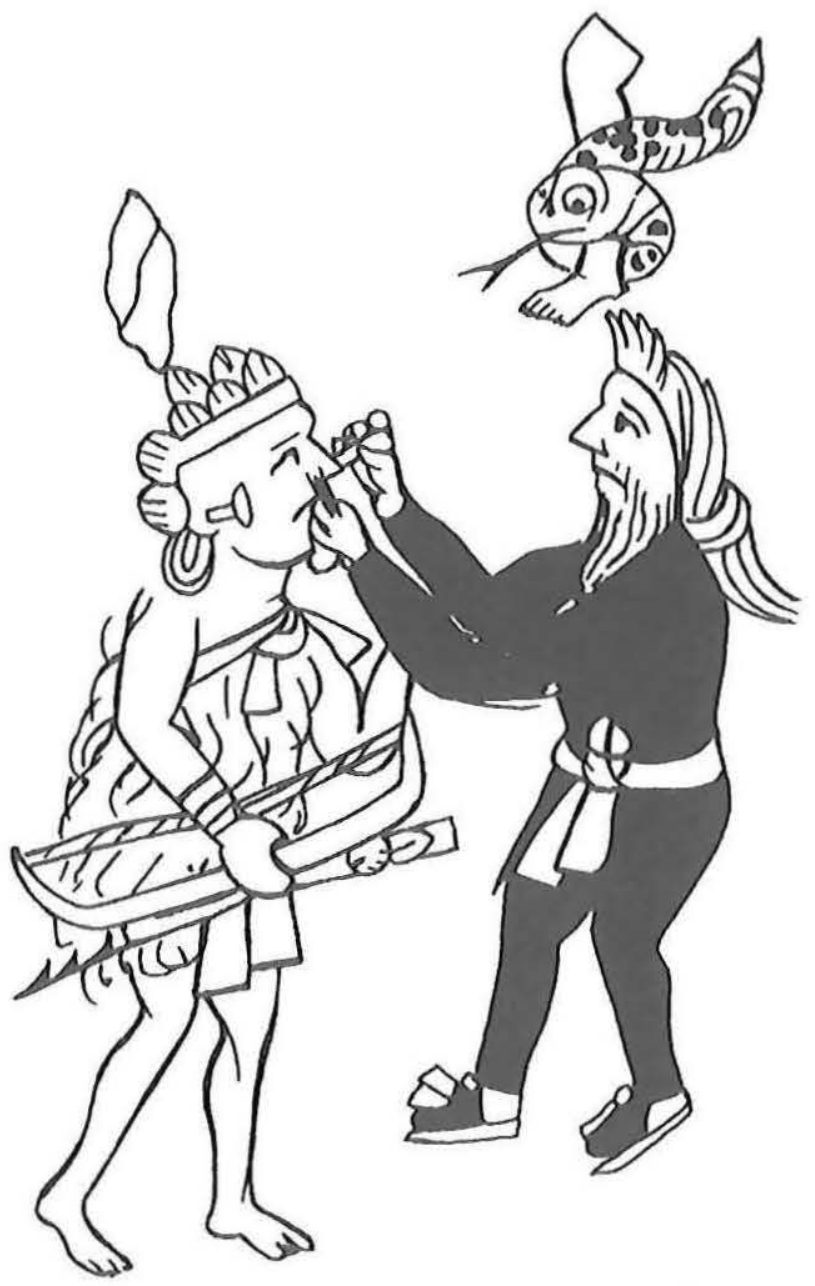

FIG. 6- Percement de la cloison nasale des tlatoque chichimèques (d'après Historia tolteca-chichimeca 1976, fol. 21r).

de manière métonymique l'ensemble des rites d'intronisation, comme le manifestent les codex mixtèques (Codex Becker I 1996, pl. 15 ; Codex Bodley 1960, pl. 9 ; Codex Colombino 1996, pl. 13) (Figure 7).

Pour expliquer la signification du percement de la cloison nasale, on peut évoquer le statut particulier des Huaxtèques, représentés avec une perforation exagérée de cette cloison, qui constituaient les victimes sacrificielles par excellence des Mexica (Durán 1995, I, p. 390). De fait, le nom que les Nahua 


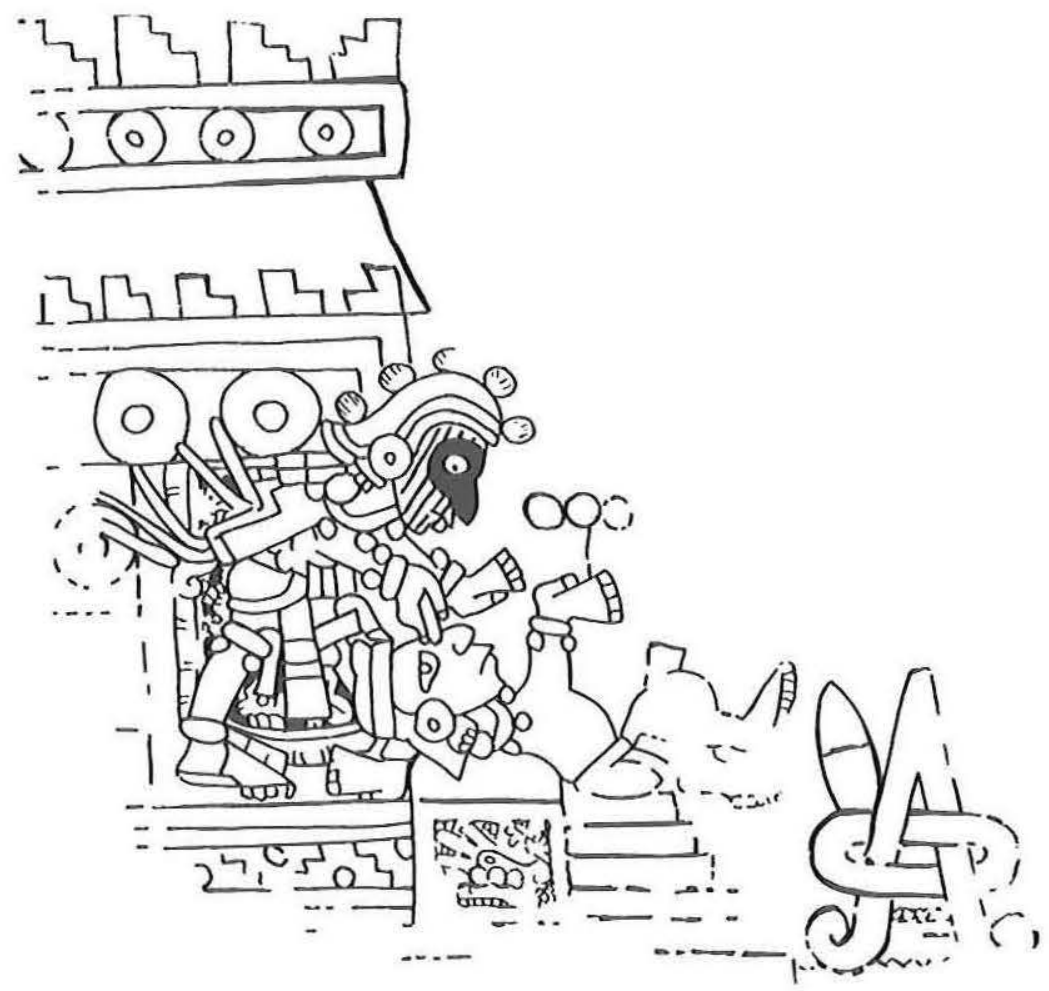

Fig. 7 - Cérémonie de percement de la cloison nasale de 8 Cerf Griffe de jaguar (d'après Codex Colombino 1996, pl. 13).

attribuaient aux Huaxtèques, tohuenyo, signifierait «ce qui constitue notre offrande », c'est-à-dire la victime de sacrifice (León-Portilla 1959, p. 111) ${ }^{34}$. Il n'est pas indifférent que des divinités liées au roi et au sacrifice comme Tezcatlipoca et Mixcoatl puissent se manifester comme des Huaxtèques (Codex Fejérváry-Mayer 1901-1902, pl. 26 ; CF, III, pp. 19-22) (Figure 8). Ils se présentent alors avec le sexe découvert, autre caractéristique commune aux Huaxtèques et aux futurs sacrifiés ${ }^{35}$. Il est peut-être significatif que les cerfs sacrifiés dans les codex apparaissent également avec le sexe apparent, ce qui est exceptionnel dans les représentations animales (Codex Borgia 1963, pl. 22). Autre élément à verser au dossier, à partir du Paradigma apologético y noticia de la lengua Huasteca de Carlos de Tapia Zenteno (1985), Ruz (1996, p. 103) a proposé l'étymologie suivante pour l'ethnonyme des Huaxtèques : 


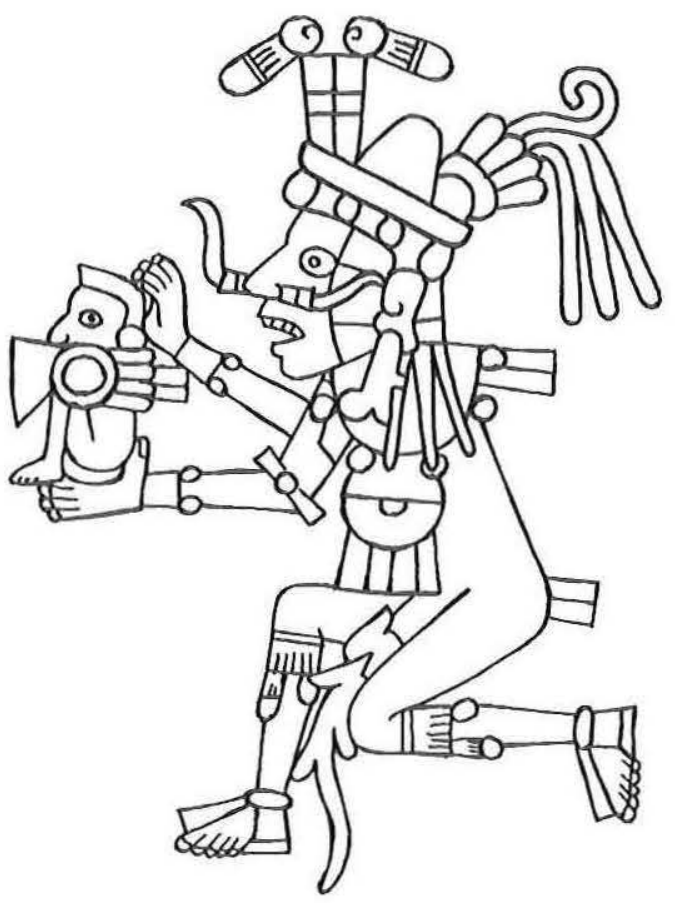

FIG. 8 - Mixcoatl représenté comme un Huaxtèque (d'après Codex Féjérvary-Mayer 1901-1902, pl. 26).

[... ] en su nombre [del venado], tenec bichim, los huaxtecos exhiben junto con el vocablo para " bestia » (bichim), nada menos que el tenec que según Tapia designa al " indio ». De tratarse de la misma raiz - lo cual parece bastante probable -, los huaxtecos compartirian con sus vecinos quejaches del sureste campechano (los "mazatecos 》 de Cortés) una significativa identificación con el venado.

Il y aurait donc des rapports étroits entre le peuple huaxtèque, la nudité, la cloison nasale percée, le cerf et le destin de sacrifié, éléments que nous trouvons également présents chez le dieu Mixcoatl, "Serpent de Nuage » (Olivier 2001, pp. 39-41). Rappelons que le futur souverain, tout comme les candidats à la noblesse, était dénudé au début des rituels (CF, VIII, p. 62; Codex Magliabechiano 1996, fol. 70r). Nous considérons que cette nudité - outre le fait qu'elle pourrait manifester la condition de "nouveau-né » du roi ou du noble sur le point d'acquérir un nouveau statut - le qualifie comme future victime, ainsi l'attestent plusieurs représentations de prisonniers nus sur le point d'être sacrifiés dans toute la Mésoamérique (Olivier 2007, pp. 235-237). Dans un ouvrage récent, Dehouve (2006b, p. 92) a proposé que les dirigeants politiques actuels, tout comme l'ancien 
tlatoani mexica, étaient assimilés à des victimes expiatoires au début des rituels et qu'il convenait de les purifier. Un écho de ces conceptions apparaît chez les Tzotzil de Chamula (Chiapas) qui utilisent l'expression " cazar víctimas » pour signifier la recherche de nouvelles autorités (Pozas 1959, p. 137). Aurore Monod Becquelin et Alain Breton (2002, p. 36) ont signalé le même phénomène chez les Tzeltal de Bachajón, « [...] le terme employé signifie vraiment "chasser", "poursuivre" $[\ldots])^{36}$.

Pour confirmer de façon définitive le symbolisme sacrificiel des rites de percement de la cloison nasale dans l'ancien Mexique, précisons que cette cérémonie, illustrée dans plusieurs codex mixtèques (Codex Becker I 1996, pl. 15 ; Codex Bodley 1960, pl. 9 ; Codex Colombino 1966, pl. 13), pouvait avoir lieu sur une pierre de sacrifice où le futur roi était adossé, voire étendu, à l'image des victimes du sacrifice humain (Figure 7) ${ }^{37}$. Ils apparaissaient ainsi à la manière des Chichimèques, allongés sur les arbustes épineux dans l'Historia toltecachichimeca $\left(1976\right.$, fol. $\left.20 \mathrm{r}^{\circ}\right)$, ou encore à l'instar des Mimixcoa sacrifiés, eux aussi étendus sur des arbustes épineux et des cactus, par les Mexica durant leur migration (Codex Boturini 1964, pl. 4) (Figure 4).

Outre le Mexique central et la zone mixtèque, cette importante cérémonie est attestée au Guatemala pour les dirigeants cakchiquel et quiché. La description la plus détaillée apparaît dans El Título de Totonicapán (1983, pp. 195-196) où il est spécifié que le rituel avait lieu pendant la grande danse en l'honneur de Tohil, divinité tutélaire des Quiché :

Los señores que tenían dos palios fueron perforados y cortados con: las piedras negras y amarillas, el saca-sangre, el palo rojo, la cola del venado, el brazalete, las garras de águila y jaguar, los caracoles, la red de tabaco, las plumas de garza y las varias piedras preciosas. Usaron las piedras preciosas para cerrar las narices de los Ajpop y Ajpop C'amjá, quienes fueron abiertos y perforados aqui en Q'uiché [...].

Cette source exceptionnelle précise que cette cérémonie avait lieu durant la vingtaine de $t$ 'iquín $k^{\prime} i j$. Or, élément d'extrême importance, Carmack et Mondloch (in El Título de Totonicapám, p. 17) signalent que cette vingtaine était équivalente à celle de quecholli au Mexique central. Nous avons vu qu'il s'agissait de la fête principale de Mixcoatl, caractérisée par le sacrifice conjoint de cerfs et de victimes humaines. L'équivalence des deux vingtaines constitue un solide argument permettant d'identifier Tohil à Mixcoatl, tous deux ayant de surcroît le cerf comme double principal (Popol Vuh 1985, p. 185) ${ }^{38}$. Nous retrouvons donc dans les Hautes Terres du Guatemala les mêmes connotations sacrificielles de la cérémonie du percement de la cloison nasale des nouveaux dirigeants ainsi qu'un lien étroit avec une divinité tutélaire dont le double est le cerf ${ }^{39}$. 


\section{Perspectives de ReCherChe}

Nous avons vu que le futur souverain, lors des rites d'intronisation, s'identifiait à un moment donné à une victime sacrificielle, dont les Huaxtèques et/ou les Mimixcoa, eux-mêmes associés à des cervidés, constituaient les modèles. On songe au processus d'intégration des ennemis (ou des " autres » en général) dans la conformation de la notion de personne mise en évidence en Amazonie par Eduardo Viveiros de Castro (1993). Dans le cas mexica, sans nier la prégnance d'un modèle mythique ancien ${ }^{40}$, on soupçonne que le choix des Mimixcoa pourrait être en rapport avec les ennemis traditionnels de la Triple Alliance, c'est-à-dire les peuples de la vallée de Puebla dont la divinité tutélaire était Camaxtli-Mixcoatl ${ }^{41}$. Quant aux Huaxtèques, outre les guerres fréquentes dont ils étaient l'objet, les traditions "mythico-historiques » des Mexica leur assignaient également une fonction originelle d'ennemis. Ne disait-on pas que le départ des Mexica d'Aztlan, leur patrie originelle, avait été motivé par l'opposition brutale entre deux frères, l'un dirigeant les Mexica et l'autre étant le tlatoani des Cuexteca, c'est-à-dire des Huaxtèques (Chimalpain 1991, pp. 20-21).

Ces données nous renvoient enfin aux liens éventuels entre la royauté sacrée et les ancêtres, analysés dans l'aire maya (McAnany 1995), guère abordés en revanche au Mexique central. Quoi qu'il en soit, les sources insistent sur le fait que le tlatoani ne faisait qu'occuper le trône délaissé par ses prédécesseurs, qualifiés d'ancêtres. Il se devait de suivre leur exemple, à l'image d'un grand arbre (pochotl, mot nahuatl qui signifie aussi " père, mère, gouvernant ») à l'ombre duquel ses sujets seraient protégés. Incarnation des souverains défunts, le nouveau roi était considéré comme « le père et la mère » du peuple qu'il gouvernait ( $\mathrm{CF}, \mathrm{VI}, \mathrm{p} .22$; Alvarado Tezozómoc 1878, pp. 239 439, 460 ; Durán 1995, I, pp. 118-119 ; etc.). Certes, on a souligné l'absence de divinisation des souverains défunts chez les Mexica ainsi que l'inexistence d'un culte dynastique (Graulich 1998). Cela étant, le rôle de Mixcoatl et des cervidés dans les rites d'accès au pouvoir pourrait traduire la volonté mexica d'intégrer des figures ancestrales dans un processus de légitimation de leur pouvoir fondé, non pas sur une origine locale ancestrale, mais essentiellement sur la conquête de peuples autochtones.

Je reconnais que cette hypothèse est fragile, mais l'insistance des sources à associer Mixcoatl et les cervidés avec les ancêtres est remarquable. En effet, plusieurs peuples de l'ancien Mexique central comme les Mazahua, les Acolhua, les Huexotla ou les habitants de Tepeticpac s'attribuaient un ancêtre nommé Mazatl, "Cerf » ${ }^{42}$. De même, Mixcoamazatzin, "Cerf Mixcoatl », aurait été le fondateur et le premier souverain de la prestigieuse cité de Tollan (Anales de Cuauhtitlan 1992a, p. 5 ; 1992b, p. 26). Enfin, Motolinía (1971, p. 10) décrit :

[... ] un anciano viejo de que ellos toman principio, llamado por nombre Iztacmixcoatlh. Éste de su mujer llamado Ilancue [Ilancueitl] hobo seis hijos. Al primero llamaron 
Gelhua; al segundo Tenuch, al tercero Ulmecatlh, al cuarto Xictlancatl, al quinto Mixtecatlh, al sexto Otomitlh; de éstos proceden grandes generaciones, casi como se lee de los hijos de Noé.

Iztac Mixcoatl, « Blanc Serpent de Nuage », apparaît donc clairement comme l'ancêtre par excellence, véritable « père des peuples » de l'ancien Mexique ${ }^{43}$. De même, il est sans doute significatif que les ancêtres de l'humanité, Cipactonal et Oxomoco, apparaisssent surmontés de bâtons à tête de cerf (Codex Borbonicus 1991, pl. 21) ${ }^{44}$. Quant à la divinité suprême, double, des anciens Mixtèques, elle était nommée " 1 Cerf Serpent de Lion » et « 1 Cerf Serpent de Tigre » (Codex Vindobonensis 1992, pl. 51 ; The Selden Roll 1955; García 1981, p. 327) (Figure 9).

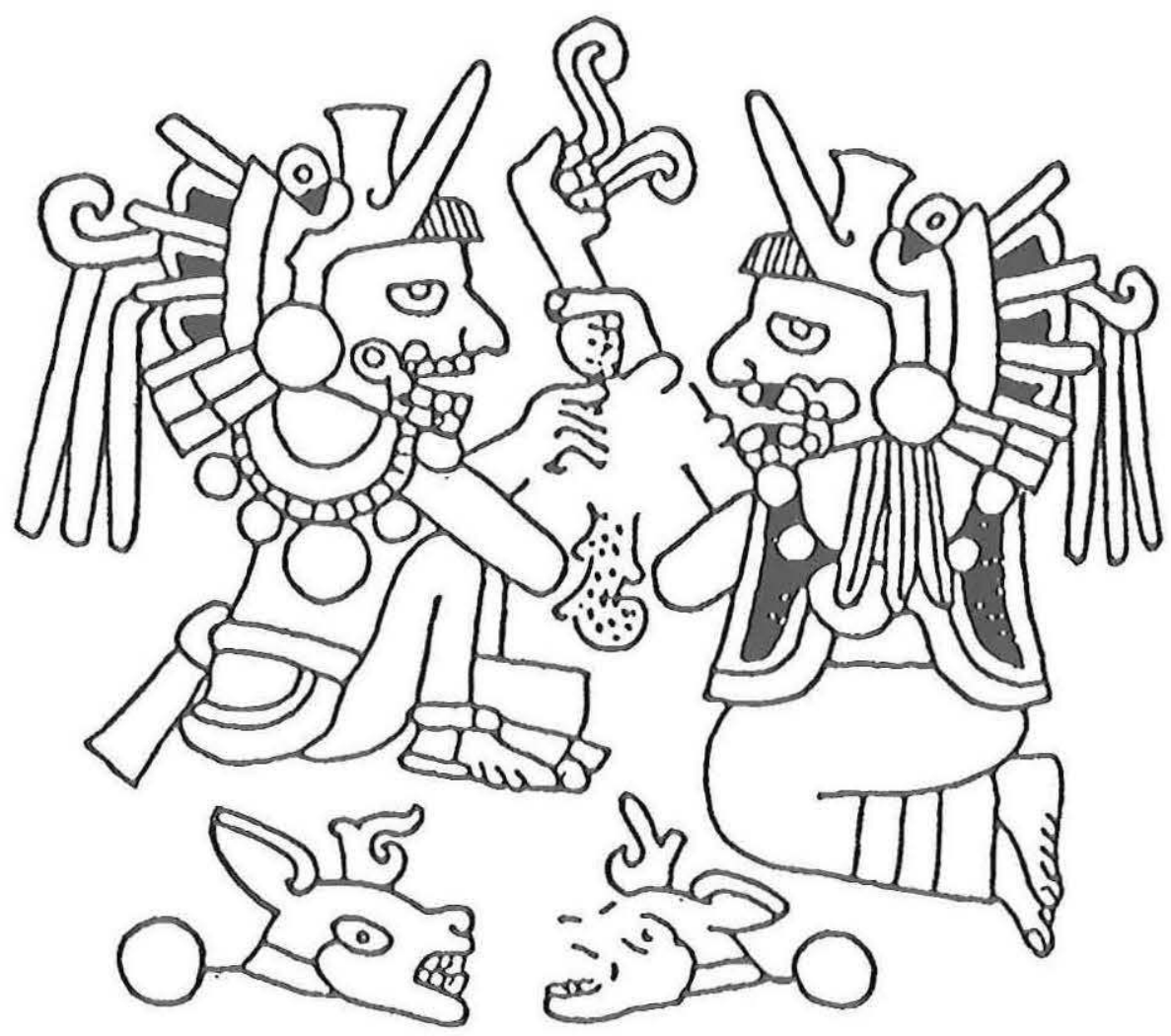

Fig. 9 - " 1 Cerf Serpent de Lion " et " 1 Cerf Serpent de Tigre ", couple suprême des anciens Mixtèques (d'après Codex Vindobonensis 1992, pl. 51). 
Nul doute qu'il conviendrait d'affiner ces analyses. Outre le cerf-victime sacrificielle, le tlatoani mexica était sucessivement identifié à plusieurs divinités durant son intronisation et il épousait les traits à la fois du sacrifiant et du sacrifié, deux états qui se confondaient, nous l'avons vu (Olivier 2008, pp. 275-276). Quant aux animaux auxquels le souverain était assimilé, n'oublions pas le jaguar, modèle du guerrier conquérant mais aussi du sorcier; ce qui le rapproche de certains " rois sorciers " africains identifiés au léopard, étudiés par Alfred Adler (2001-2002). De nos jours, dans certaines communautés indigènes, c'est le dindon ou le poulet qui constituent les prototypes des animaux sacrifiés; de ce fait,

[... ] en su atuendo, los funcionarios de Zinacantan se parecen a los pollos, cuyas plumas negras y barbas y crestas rojas combinan esos colores en forma similar: Además la palabra tzotzil para los funcionarios y los pollos sacrificiales es la misma: K'exoliletik. Los funcionarios son sustitutos de los dioses ancestrales que en el pasado mitológico ocuparon sus cargos. Los pollos sacrificiales funcionan como sustitutos o reemplazantes de la vida del paciente al ser ofrecidos a los dioses. El funcionario sacrifica tiempo, energia y recursos por los dioses y en beneficio de la commidad. (Vogt 1979, p. 135)

À travers cette analyse de Evon Z. Vogt (ibid.), nous retrouvons la métaphore du sacrifice comme fondement de l'autorité politique, autorité étroitement liée aux divinités ancestrales. À l'époque précolombienne, confondu avec l'image de l'ancêtre, le cerf représentait sans doute l'un des modèles sacrificiels auquel le souverain pouvait s'identifier, père de ses sujets mais aussi sacrifié par excellence, afin d'assurer leur existence et celle de l'univers ${ }^{45}$. *

* Manuscrit reçu en janvier 2007 et accepté pour publication en janvier 2008.

\section{Notes}

Je tiens à remercier vivement Michel Graulich pour ses commentaires, Alain Breton et Grégory Pereira pour leur invitation à participer à ce dossier et Rodolfo Ávila qui a réalisé les dessins qui illustrent cet article.

1. J'utilise par commodité le terme « cerf ». En fait, les cervidés présents sur les territoires mexicain et centraméricain ont été classifiés par les spécialistes en cinq espèces : une sous-espèce du grand cerf nord-américain ou wapiti aujourd'hui disparue (Cervus canadiensis merriani) et le cerf mule ou à queue noire (Odocoileus hemionus), habitants des zones septentrionales arides du Mexique; le cerf à queue blanche (Odocoileus virginiamus), présent sur tout le territoire mésoaméricain (en fait, la plupart de nos données le concerne) ; le temazate rouge ou chevreuil (Mazama americana) qui habite les forêts tropicales de la Côte du Golfe du Yucatan et en général le sud-est et une partie du sud-ouest du Mexique ; enfin, le temazate gris (Mazama pandora) que l'on rencontre dans les forêts des États du Chiapas, Campeche et Quintana Roo (Leopold 1959, pp. 501-523).

2. On consultera la Relación geográfica de Dzonot (in Relaciones histórico-geográficas de la gobernación de Yucatán 1983, II, p. 86), Balsalobre (1987, p. 250), Wagley (1957, p. 210), Schultze-Jena (1946, p. 35), etc.

3. Parmi de nombreuses références, voir Arlegui (1851, pp. 146-147), Berlin (1957, p. 21), Preuss (1998, p. 282), Chapman (1970, p. 58), Redfield et Villa Rojas (1934, p. 140), etc. Il existe une 
abondante documentation sur les bézoards : voir Pomar (1986, p. 111), Hernández (1959, III, pp. 307309), García de León (1969, p. 308), Chapman (1985, p. 147), Hémond (1996, pp. 276-277), etc.

4. Nous devons à Karl Anton Nowotny (2005 [1961], pp. 302-306) la découverte de la signification de cette section du Codex Cospi. Sur le livret chontal, voir Carrasco (1960, pp. 96-98).

5. Quelques données sur ces rêves prémonitoires se trouvent dans Wisdom (1961, p. 96), Zingg (1982, I, p. 456) et Hill (1938, pp. 98, 108-109, 114, 118). Sur les rêves érotiques, voir Braakhuis (2001, p. 392).

6. Pour cette raison sans doute, les Huichol du début du $x^{\mathrm{e}}$ siècle déclarèrent à Lumholtz (1986, II, p. 40) que les pièges devaient être posés par des gens «au cœur pur ", celui placé par un chasseur amoureux serait détecté par le cerf qui s'enfuierait immédiatement.

7. Il s'agit en réalité d'une " cosmovision cynégétique » très proche de celle des chasseurs sibériens. Ainsi, le chamane toungouse prend l'aspect d'un renne femelle pour attirer vers les chasseurs ses congénères. De même, les Youkaghir affirment que, si le renne n'est pas amoureux du chasseur, il ne se laissera pas tuer (Lot-Falk 1953, pp. 55-56, 76). Les analyses de Roberte Hamayon (1998) sont particulièrement éclairantes à cet égard ; elle considère que les peuples sibériens assimilent la chasse à un échange entre les hommes et les esprits des animaux. Une alliance métaphorique s'établit entre une épouse cervidée - proie par excellence - et le chamane dont la fonction est de faciliter la capture des animaux. De ce fait, le chamane " s'animalise » durant le rituel, revêt une peau de cerf et ses bois, imite le cervidé qui combat et s'accouple. À la fin du rituel, le chamane s'écroule et se transforme en proie, dévorée dit-on par les esprits de la forêt, comme le cerf auquel il s'est identifié. De cette façon, il est censé restituer le gibier abattu par les chasseurs.

8. J'ai réalisé moi-même les traductions des textes en langue nahuatl cités dans cet article.

9. Afin de ne pas multiplier les références, nous signalerons seulement les entrées du vocabulaire nahuatl de Molina $\left(1970\right.$, fol. $\left.22 \mathrm{v}^{\circ}\right)$, les données linguistiques mayas réunies par Ruz (1996, p. 95) et le mythe huichol recueilli par Zingg (1982, I, p. 547).

10. Des chasseurs déguisés en cerf sont représentés sur des céramiques maya (Reents Budet 1994, p. 263). Cette technique cynégétique est mentionnée par José Arlegui (1851, p. 169) à propos des Indiens de l'État de Zacatecas au début du XvII' siècle. Enfin, nous disposons d'une remarquable description de l'élaboration et de l'utilisation d'un déguisement de cerf par les Navajo dans les années 1930 (Hill 1938, pp. 123-126).

11. Précisons toutefois que les chasseurs bredouilles pouvaient réagir de manière violente aux dépens d'une divinité peu coopératrice, par exemple au Yucatan : " [...] un dios que decían que era venado; y si no mataba algo aquel dia ibase a su casa aquel indio, le quebraba y dábale de coces diciendo que no era buen dios » (Relaciones histórico-geográficas de la gobernación de Yucatán 1983, p. 286).

12. À propos de cette importante conception, voir les réflexions de Preuss (1998, pp. 191, 278-279) et Neurath (2002, pp. 98, 175, 191-192).

13. Parmi de nombreuses références, on peut consulter les passages des auvres suivantes: Landa (1959, pp. 74-75), Balsalobre (1987, p. 250), Lumholtz (1986, II, p. 45), Zingg (1982, I, p. 457), Hill (1938, p. 110), Furst (1972, pp. 53, 86-87), Myerhoff (1974, p. 174), Neff Nuixa (2001, p. 367), Chapman (1978, p. 132), Dehouve (2006a, p. 199), etc.

14. Au sujet des Triqui, les données nous ont été fournies par le maestro Antonio Ramírez Silva durant un court séjour à Yosoyusi (Oaxaca). Je le remercie vivement, ainsi que Swanilda Mendoza González qui nous invita à cette occasion.

15. Les chasseurs nahua de Pajapán conservent également les mâchoires inféricures des cerfs chassés, mais l'ethnologue ne précise pas, hélas, l'endroit où elles étaient déposées (Garcia de León 1969, p. 308).

16. Voir les témoignages d'Alva Ixtlilxóchitl (1985, II, p. 157) et d'Alvarado Tezozómoc (1878, pp. 323, 517), ainsi que les études de López Luján (1993, pp. 262-270) et Graulich (2005, p. 321).

17. Des découvertes similaires - personnages sacrifiés associés à des maxillaires en coquillage - ont été effectuées durant les fouilles récentes de la pyramide de la Lune dans la même métropole (Sugiyama et López Luján 2007, pp. 130, 142, 144). 
18. Par exemple, les Huichol brûlent les os des cerfs " [...] ya que se cree que Tatewari /le dieu du Feu] hará que renazca un nuevo venado de las cenizas del esqueleto " (Furst 1972, p. 90 ; Myerhoff 1974, p. 175).

19. L'ethnologue française explique que « [...] el algodón en otros rituales de la zona, está asociado con la humedad, y en este caso con la ropa, que ocupa el lugar de la carne y de la piel, partes húmedas del animal ".

20. Plusieurs divinités à la mâchoire inférieure décharnée ont été représentées dans les codex mixtèques. Parmi elles, Jill Leslie Furst (1982) signale le couple suprême du panthéon mixtèque ( 1 Cerf " et " 1 Cerf ») (Figure 9) ainsi que des déesses associées au vin d'agave (" 2 Fleur » et " 3 Crocodile »), à la terre et au maïs ( 9 Herbe »). Cet auteur considère que ces divinités, pourvoyeuses de vie et de subsistances, arborent ce type de maxillaire inférieur de par le pouvoir de régénération attribué aux os en Mésoamérique.

21. Le commentaire d'un mara'akame huichol pourrait renforcer cette hypothèse. En effet, après avoir récolté ( « chassé ») le peyotl, équivalent du cerf, on doit laisser dans le sol quelques racines de cette plante hallucinogène, " [...] so that the deer could grow again from his bones " (Myerhoff 1974, p. 155).

22. Enfin, un parallèle peut être établi avec les pratiques funéraires des chasseurs sibériens qui concernent également leurs prises de chasse (Hamayon 2001a). En effet, de même que les défunts sont placés sur des plates-formes en bois reposant sur quatre piliers dans la forêt, les chasseurs réalisent des opérations rituelles similaires avec les cerfs et les ours chassés. Toutefois, dans ce dernier cas, ils ne déposent que les crânes et parfois les os longs sur des planches inclinées, adossées à des arbres dont la partie supérieure a été coupée. Parfois, les crânes et les mâchoires sont disposés sur des branches d'arbres. Il s'agit, que ce soit pour les humains ou les animaux, de nier la mort comme terme absolu de la vie et d'intégrer chaque défunt individuel dans un processus symbolique global qui fait de la mort une étape indispensable du cycle de vie. Hamayon (ibid., p. 113) ajoute que ce traitement funéraire a pour but de " [...] libérer la chair pour la rendre consommable par autrui et permettre à l'os de servir de support symbolique à un nouvel être de même lignée ou de même espèce [...] " .

23. Les matériaux manquent pour parvenir à des analyses plus fines. Examinant les pratiques cynégétiques et guerrières des Huaulu d’Indonésie, Valerio Valeri (1994, p. 129) a pu observer des similitudes, mais aussi des différences subtiles entre le traitement des animaux chassés et des ennemis tués : As I have indicated, the head of deer and wild pig is left unprocessed where the killing has occurred, that is, in the forest. The carcass is instead transformed and incorporated into the village for food. Just the opposite happens with human victims. The carcass is left behind, untouched, and the head is brought into the village, transformed and incorporated there as the "food" of the "masters of the house" spirits. There is, however, a partial overlapping between animal and human head if we take into account that the lower jaw of the animal is treated in a way that is reminiscent of the way the human head is treated. Indeed, the jaw is brought back to the village, left on the shelf over the fireplace of the house of the humter, and then hung under the roof, just like the human heads in the temple, for display' ".

24. En effet, l'Historia de los mexicanos por sus pinturas (1941, p. 216) précise que Camaxtli créa cinq Mimixcoa au huitième ciel. Or, selon fray Andrés de Olmos (in Thévet 1905, p. 22), il s'agit du niveau céleste où réside Tlaloc. En outre, on lit dans la Leyenda de los Soles (1992a, p. 91 ; 1992b, p. 150) que Iztac Chalchiuhtlicue, épouse de Tlaloc, fut la mère des Mimixcoa. Ce lieu de naissance pourra sembler étrange, mais n'oublions pas que les «Serpents de Nuage » sont, comme leur nom l'indique, associés à la pluie et à la foudre. On retrouve des caractéristiques semblables avec les cervidés (Olivier 1999-2000, p. 86). Signalons simplement que Tlaloc est le patron du jour mazatl (Codex Borgia 1963, pl. 12 ; Codex Vaticamus 3773 1902-1903, pl. 31).

25. L'équivalence entre l'activité guerrière et la pratique de la chasse est illustrée par la description de la fète de quecholli parmi les habitants de la cité d'Acolman : " [...] el dia de la fiesta por la mañana, juntarse todos los macehuales y, puestos en hábitos de guerra, ir a cazar [...] " (Relación geográfica de Acolman in Relaciones geográficas del siglo xv: México 1986, p. 229).

26. Provenant de l'aire maya, la peinture du Scaffold Vase conservé à Dumbarton Oaks (Washington DC) représente un homme à quatre pattes, comme un cerf, sur le point d'être sacrifié sur un cadre 
en bois. Un personnage en céramique provenant de l'ile de Jaina adopte la même attitude, en outre il porte un heaume de cerf (Taube 1988, pp. 333-335).

27. En langue nahuatl, les mêmes verbes tlamalia, axilia et amilia, signifient " Caçar hombres o aues para otros ", ce qui pourrait traduire une équivalence entre la chasse et la guerre (Molina 1970, fol. $22 \mathrm{v})$.

28. Dans sa traduction du Chilam Balam de Chumayel (1973, pp. 76-77), Ralph Roys a signalé que le mot ah-cehob était utilisé pour désigner les chasseurs. Karl Taube (1988, p. 334) précise que ce mot designait les "chasseurs de cerf " (ceh signifie "cerf " en maya yucatèque) et que, dans le contexte guerrier de ce passage du Chilam Balam de Chumayel, "[...] the executioners were identified as deer-slayers and the prisoners as their prey " .

29. "Las flechas son de un carrizo delgado y duro amarradas en sus extremidades con unos nervios de venado tan bien puestos que no hay mudos ni se ve donde acaba la ligadura o donde empieza " (León 1961, p. 37). Voir également la Relación geográfica de Ameca in Relaciones geográficas del siglo xvt: Nueva Galicia (1988, p. 38).

30. Citons le passage de Motolinía (1971, p. 307) : « [...] y si moria en la guerra, o podian cobrar el cuerpo o no, si podian haber el cuerpo, allá lo quemaban sin ceremonias, e cuando volvían a su tierra traían una saeta de aquel que ya habian quemado, y dábanla a los de su casa, y ellos la componian y ataviaban, y la tenían por imagen del muerto, y vestida de las insignias del sol, quemábanla ».

31. Chez les Chumash, ces prohibitions s'étendaient à tous les animaux chassés, y compris les poissons (Jackson 2002, p. 83).

32. Frederick Starr (1900, p. 55), qui déclare suivre les données du naturaliste nord-américain E. W. Nelson, écrit : " [...] at San Juan Guichocovi he says there is a great rock on which young [Mixes] humters must place the skull of the first game they kill: they must under no circunstances eat the meat of these animals 》.

33. Muñoz Camargo (1998, pp. 85-86) mentionne l'adoption d'un nouveau nom pour les guerriers tlaxcaltèques qui « se armaron caballeros », c'est-à-dire qui accédaient à la noblesse. Des déchiffrements récents de glyphes mayas révèlent qu'à l'époque Classique les rois mayas changeaient également de nom lors de leur intronisation (Grube 2001, pp. 72-73).

34. D'après Michel Launey (1980, II, 255), «Tohueyo : formation peu claire ; on trouve parfois tohuenyo, qui est peut-être la forme exacte : faut-il alors comprendre "notre offrande" (huentli) ??? ou voir une forme dialectale dans laquelle -huen- serait une variante de -huan-? "

35. En outre, Tezcatlipoca en tant que tohuenyo, nu, se présente clairement comme une future victime. De fait, il exige une mort sacrificielle auprès du roi Huemac (CF, III, p. 20). Ce dernier le marie à sa fille au lieu de le sacrifier, commettant ainsi une transgression qui précipite la ruine de Tollan (Olivier 1997, pp. 175-179).

36. Pour expliquer cet étrange statut de " victimes sacrificielles » attribué aux gouvernants, il faut revenir sur le texte qui décrit le sacrifice des Mimixcoa par les Mexica. À leur sujet, Huitzilopochtli affirme " [...] yehuantin y acachto tequitizque », que Victor Castillo (in Chimalpain 1997, p. 15) traduit comme " Éstos serán los primeros que asuman el trabajo ». Le verbe tequitia, "dar obra o tequio a otro » (Molina 1970, fol. $105 \mathrm{r}^{\circ}$ ), est utilisé pour exprimer la fonction assignée aux Mimixcoa, c'est-à-dire leur rôle de victimes sacrificielles. Être sacrifié correspond donc à un " travail », une " charge " - tequitia signifie aussi " encargarse de algo " (ibid.) - ce qui coincide avec la fonction attribuée aux gouvernants. Nous retrouvons un concept similaire - qui implique le sacrifice potentiel du roi comparé à celui d'une divinité - dans l'Occident médiéval. Selon Ernst Kantorowicz (2000, p. 839), « [...]le Prince étant la tête du corps mystique de l'État, et quelquefois ce corps lui-même, était comparable au Christ, qui était à la fois la tête et le corps mystique de l'Église et ce corps lui-même ; d'autre part, tout comme le Christ avait donné sa vie pour son corps corporatif, le Prince était censé sacrifier sa vie pour le bien commun ".

37. Ajoutons que, dans plusieurs de ces manuscrits, le personnage qui effectue le percement de la cloison nasale porte des attributs caractéristiques de Mixcoatl, peinture noire autour des yeux et/ou coiffe avec des boules de duvet ainsi que des rayures blanches et rouges sur le visage (Codex Becker I 1996, pl. 15; Codex Bodley 1960, pl.9 ; Codex Colombino 1996, pl. 13). Il conviendrait d'expliquer 
pourquoi, dans ce cas, c'est le sacrificateur et non pas le « futur souverain-victime ", qui apparaît sous les traits de Mixcoatl ou d'un Mimixcoa. Rappelons que, dans les manuscrits mixtèques, les Toltèques ou Toltèques-Chichimèques sont représentés de cette façon, illustrant ainsi le mot mixtèque "sami nuu " qui les désigne et qui signifie " brûle l'œil » ou "brûle la face ". Ainsi, on considère que le personnage appelé 4 Jaguar - dont l'identité suscite encore des polémiques - qui perce la cloison nasale de rois tels que 8 Cerf Grifle de Jaguar ou 4 Vent provient du Mexique central (Jansen 1989, pp. 71-72).

38. Il n'est pas indifférent de retrouver, parmi les cervidés décrits par les informateurs de Sahagún, l'izıac maşatl ( " cerf blanc ») qualifié de " [...] roi des cerfs (/...] intlatocauh in mamą̧a) » (CF, XI, p. 15). Hernández (1959, III, p. 307) signale également des « [... ] ciervos completamente blancos, que los indios creen reyes de los ciervos y llaman por su color iztacmazame ». De plus, la couleur blanche est étroitement associée à l'idée d'origine, comme l'exprime, par exemple, la blancheur attribuée au site originel Aztlan (Durán 1995, I, p. 71).

39. Pourrait-on appliquer ces éléments d'interprétation au Monument 1 , récemment mis au jour à El Kinel (Chiapas) ? Sur cette stèle (ou ce linteau, les auteurs s'interrogent à cet égard) apparaît le roi de Yaxchilan, Bouclier Jaguar III, en train de danser vêtu comme un captif. En outre, il porte un splendide heaume de cerf ! (Houston et al. 2006, pp. 91-92).

40. En effet, le concept de Mimixcoa comme victimes sacrificielles se retrouve aussi bien dans les manuscrits du groupe Borgia que dans les manuscrits mixtèques. En outre, nous avons proposé qu'il existait à Cacaxtla une représentation de Mimixcoa de l'époque Épiclassique (Olivier 2001, pp. 40-41).

41. Dans un de ses premiers livres, Claude Nigel Davies (1968, pp. 215-217) avait mis l'accent sur cette singularité : " [...] tenemos un curioso semicírculo de pueblos, desde Metztitlan hasta Yopitzinco, y quizá hasta Tototepec del Sur, que se distingue de otros pueblos por no haberse sometido al Imperio Azteca, y por poseer ciertas deidades en comín, todas ligadas con Mixcoatl [...] ".

42. Lienzos y Códice de Tepeticpac (1986); Sahagún (1993, fol. 53r $\mathrm{r}^{\circ}$; CF, X, p. 184 ; Anales de Tlatelolco (1980, p. 31). La littérature ethnographique offre des exemples similaires, comme chez les Popoloca de San Felipe Otlatepec (Puebla) dont le héros culturel et ancêtre fondateur, Don Gonzalo Mazatzin, serait le fils d'une femme et d'un cerf (Jäcklein 1974, pp. 34-35). D'après Jon Christopher Crocker (1985, p. 163), "The Bororo [du Brésil] collectively term themselves "the children of pogodo" (brocket deer), since in one origin myth a Bororo man mates with a female deer of this species to repopulate the earth after everyone but him has drowned in a flood "). De même, les Candoshi d'Équateur considèrent le cerf comme un ancêtre (Surrallés 2003, pp. 112-113).

43. Un autre indice apparaît à travers la description d'un cervidé appelé tlamacazcamaçatl (« cerf prêtre ») : « [...] il est très gros, très grand, orné, les trous [contours] de ses yeux peints en noir [...] (vel veiac vel quauhtic : mjhichiuh, mjhixtetlilcomolo [...]) " (CF, XI, p. 15). La même expression, mixtetlilcomolo, est utilisée pour décrire la peinture faciale du dieu Atlahua, un avatar de Mixcoatl (Sahagún 1993 , fol. $\left.265 \mathrm{r}^{\circ}\right)$.

44. À Zinacantan (Chiapas), Victoria R. Bricker (1986, p. 29) décrit des bâtons utilisés par les anciens Tzotzil réalisés avec des os de cerf tandis que, chez les Otomi, les vieux du carnaval qui représentent les ancêtres portent des peaux de cerf (Galinier 1990, pp. 590-591).

45. Finalement, souhaitons que cette étude conduise à une réévaluation de la place centrale de la chasse dans la vision du monde mésoaméricaine (Graulich 1997 ; Dehouve 2006a). En effet, ce sont des sociétés que l'on caractérise volontiers comme agraires et où ont été soulignés - à juste titre - la prégnance des modèles agricoles et le rôle fondamental du cycle du maïs dans leurs conceptions religieuses. Si les rapports entre animaux et chasseurs, les liens entre la chasse et la guerre semblent proches, à bien des égards, de ceux décrits par les spécialistes des sociétés amazoniennes, voire sibériennes, il est pour le moins frappant de retrouver cette vision du monde au centre de l'idéologie royale mexica, mixtèque ou maya qui dominait des sociétés urbaines fortement hiérarchisćes. Faut-il y voir la valorisation d'un héritage sublimé d'ancêtres chasseurs («chichimèques " plus ou moins mythifiés) qui continue à structurer les valeurs des sociétés du Postclassique ? Sans doute, mais il conviendrait de poursuivre ces recherches en examinant les liens entre chasse et agriculture, deux activités que nous dissocions habituellement, mais qui présentent de significatifs points de 
convergences. Ainsi, l'idée selon laquelle les animaux chassés renaissaient à partir de leurs ossements assimilés à des semences participe de cette imbrication conceptuelle entre chasse et agriculture. Dernier exemple, les chasseurs otomi qui participaient à une grande battue organisée par le vice-roi Antonio de Mendoza, tuèrent les cerfs rassemblés... à l'aide de bâtons fouisseurs (coa) ! (voir Torquemada 1975, II, pp. 366-368).

\title{
RÉFÉRENCES BIBLIOGRAPHIQUES
}

\author{
ADV Akademische Druck-u. Verlagsanstalt. \\ CEM Centro de Estudios Mayas, UNAM. \\ CEMCA Centre d'Études mexicaines et centraméricaines, Mexico. \\ CF Voir Sahagún, Florentine Codex. \\ CIESAS Centro de Investigaciones y Estudios Superiores en Antropología Social. \\ CNRS Centre National de la Recherche Scientifique. \\ ECN Estudios de Cultura Náhuatl, UNAM. \\ FCE Fondo de Cultura Económica. \\ IIA Instituto de Investigaciones Antropológicas, UNAM. \\ IIF Instituto de Investigaciones Filológicas, UNAM. \\ IIH Instituto de Investigaciones Históricas, UNAM. \\ INAH Instituto Nacional de Antropología e Historia. \\ INI Instituto Nacional Indigenista. \\ JSA Journal de la Société des Américanistes. \\ SEP Secretaría de Educación Pública. \\ UNAM Universidad Nacional Autónoma de México.
}

ADLER Alfred

2001-2002 «Le roi sorcier», Annuaire de l'École Pratique des Hautes Études, 110, pp. 27-68.

Alva Ixtlilxóchitl Fernando de

1985 Obras históricas, 2 vol., éd. Edmundo O'Gorman, UNAM, IIH, Mexico.

Alvarado Solís Neyra Patricia

2004 Titailpí... timokotonal. Atar la vida, trozar la muerte. El sistema ritual de los mexicaneros de Durango, Universidad Michoacana de San Nicolás de Hidalgo, Morelia, Michoacán.

Alvarado Tezozómoc Hernando

1878 Crónica mexicana, éd. Manuel Orozco y Berra, Porrúa, Mexico.

Anales de Cuauhtitlan

1992a in John Bierhorst, Códice Chimalpopoca. The text in nahuatl with a glossary and grammatical notes, The University of Arizona Press, Tucson/Londres, pp. 3-84.

1992b in John Bierhorst, History and mythology of the Aztecs. The Codex Chimalpopoca, The University of Arizona Press, Tucson/Londres, pp. 23-138. 
Anales de Tlatelolco. Unos Anales Históricos de la Nación Mexicana y Códice de Tlatelolco 1980 éd. et trad. Heinrich Berlin et Robert Barlow, Porrúa, Mexico [1948].

Arias de SaAvedra O.F.M. Antonio

1990 "Información rendida por el P. [...] acerca del estado de la Sierra del Nayarit, en el siglo xvi » [1673], in Thomas Calvo (éd.), Los albores de um muevo mundo: siglos xVI-XvII, CEMCA, Universidad de Guadalajara, pp. 284-309.

Arlegui José

1851 Crónica de la provincia de N.S.P.S. Francisco de Zacatecas, Editorial Cumplido, Mexico [1737].

BaLsalobre Gonçalo de

1987 « Relación auténtica de las idolatrías, supersticiones, vanas observaciones de los indios del obispado de Oaxaca ", in Fernando Benítez (éd.), El alma encantada, FCE, Mexico, pp. 227-260.

BARco Miguel del

1988 Historia natural y crónica de la antigua California, éd. Miguel León-Portilla, UNAM, IIH, Mexico.

BERLIN Heinrich

1957 Las antiguas creencias en San Miguel Sola, Oaxaca, México, Hamburgischen Museums für Völkerkunde und Vorgeschichte, Hamburg.

BOREMANSE Didier

1986 Contes et mythologie des Indiens lacandons. Contribution à l'étude de la tradition orale maya, L'Harmattan, Paris.

BRAAKHUIS H. E. M.

2001 "The way of all flesh. Sexual implication of the Mayan hunt », Anthropos, 96, pp. 391-409.

BRICKER Victoria R.

1986 Humor ritual en la altiplanicie de Chiapas, FCE, Mexico [1973].

CARLSEn Robert S. et Martin Prechtel

1991 "The flowering of the dead : an interpretation of highland Maya culture », Man, 26, pp. 23-42.

Carrasco Pedro

1960 Pagan rituals and beliefs among the Chontal Indians of Oaxaca, México, University of California Press, Anthropological Records 20 (3), Berkeley/Los Angeles, California.

Cervantes de Salazar Francisco

1985 Crónica de la Nueva España, éd. Juan Miralles Ostos, Porrúa, Mexico. 
Chapman Anne

1970 «Chamanisme et magie des ficelles chez les Tolupan (Jicaque) du Honduras ", JSA, 59, pp. 43-64.

1978 Les enfants de la mort: univers mythique des Indiens Tolupan (Jicaque), Honduras, Études Mesoaméricaines, vol. 4, Mission Archéologique et Ethnologique Française au Mexique, Mexico.

1985 Los hijos del copal y de la candela. Ritos agrarios y tradición oral de los lencas de Honduras, UNAM, IIA, Mexico.

Chilam Balam de Chumayel

1973 The book of Chilam Balam of Chumayel, éd. et trad. Ralph L. Roys, University of Oklahoma Press, Norman [1933].

\section{Chimalpain Cuauhtlehuanitzin}

1991 Memorial breve acerca de la fundación de la ciudad de Cullhuacan, éd. et trad. Víctor M. Castillo F., UNAM, IIH, Mexico.

1997 Primer amoxtli libro. 3a relación de las différentes histoires originales, éd. et trad. Víctor M. Castillo F., UNAM, IIH, Mexico.

Codex Aubin

1963 éd. et trad. Charles E. Dibble, Porrúa, Mexico.

\section{Codex Becker I}

1996 in Codex Alfonso Caso. La vida de 8-Venado, Garra de Tigre (ColombinoBecker I), Patronato Indígena, AC, Mexico.

Codex Bodley

1960 éd. Alfonso Caso, Sociedad Mexicana de Antropología, Mexico.

Codex Borbonicus

1991 éd. Ferdinand Anders, Maarten Jansen et Luis Reyes García, ADV/FCE, Graz/Mexico.

Codex Borgia

19633 vol., éd. Eduard Seler, FCE, Mexico.

\section{Codex Boturini}

1964 in Lord Kinsborough, Antigüedades de México, 4 vol, éd. José Corona Núñez, Secretaría de Hacienda y Crédito Público, Mexico, 2, pp. 7-29.

\section{Codex Colombino}

1996 in Codex Alfonso Caso. La vida de 8-Venado, Garra de Tigre (ColombinoBecker I), Patronato Indígena, AC, Mexico.

\section{Codex Cospi}

1994

éd. Ferdinand Anders, Maarten Jansen et Peter van der Loo, ADV/FCE, Graz/Mexico.

Codex Fejérváry-Mayer

1901-1902 éd. Eduard Seler, Edinburgh University Press, Berlin/Londres. 


\section{Codex Florentino}

1979 El manuscrito 218-220 de la colección Palatina de la Biblioteca Medicea Laurenziana, éd. facs, 3 vol., Giunti Barbéra/Archivo General de la Nación, Mexico/Florencia.

Códice Laud

1966 MS Laud Misc. 678, Bodleian Library Oxford, éd. Cottie A. Burland, ADV, Graz.

Codex Madrid ou Codex Tro-Cortesiano

1977 in Códices mayas, J. Antonio Villacorta et Carlos A. Villacorta (éds), Tipografia nacional, Guatemala.

Codex Magliabechiano

1996 éd. Ferdinand Anders et Maarten Jansen, ADV/FCE, Graz/Mexico.

\section{Codex Nuttall}

1992 éd. Ferdinand Anders, Maarten Jansen et Gabina Aurora Pérez Jíménez, ADV/FCE, Graz/Mexico.

\section{Codex Tudela}

1980 éd. José Tudela de la Orden, Ediciones Cultura Hispánica del Instituto de Cooperación Iberoamericano, Madrid.

\section{Codex Vaticamus 3773}

1902-1903 éd. Eduard Seler, Edinburgh University Press, Berlin/Londres.

Codex Vindobonensis Mexicanus I

1992 éd. Ferdinand Anders et Maarten Jansen, ADV/FCE, Graz/Mexico.

Coto Thomás de

1983 Thesaurus Verborum. Vocabulario de la lengua Cakchiquel v(el) Guatemalteca, Nuevamente hecho y recopilado con summo estudio, travajo y erudición, éd. René Acuña, UNAM, IIF, Mexico [1650].

\section{Crocker Jon Christopher}

1985 Vital souls. Bororo cosmology, natural symbolism and shamanism, The University of Arizona Press, Tucson.

DAviEs Claude Nigel

1968 Los señorios independientes del imperio azteca, INAH, Mexico.

Dehouve Danièle

2006a "Les rituels cynégétiques des Indiens mexicains », in Isabelle Sidéra (éd.), La chasse. Pratiques sociales et symboliques, De Boccard, Paris, pp. 195-204. 2006b Essai sur la royauté sacrée en République mexicaine, CNRS Éditions, Paris.

Descola Philippe

1986 La nature domestique. Symbolisme et praxis dans l'écologie des Achuar, Éditions de la Maison des Sciences de l'Homme, Paris. 
DuRÁN Fray Diego

1995 Historia de las Indias de Nueva España e islas de Tierra Firme, 2 vol., éd. José Rubén Romero et Rosa Carmelo, Cien de México/CONACULTA, Mexico.

El Ritual de los Bacabes

1987 éd. et trad. Ramón Arzápalo, UNAM, IIA, Mexico.

El Título de Totonicapán

1983 éd. et trad. Robert M. Carmack et James L. Mondloch, UNAM, IIF, CEM, Mexico.

FASH William L.

1991 Scribes, warriors and kings. The city of Copán and the Ancient Maya, Thames and Hudson, Londres.

FERnÁNDEZ de Oviedo Gonzalo

1945 Historia general y natural de las Indias, 14 vol., Guaranía, Asunción del Paraguay.

Frazer Sir James George

1981-1984 Le Rameau d'or, 4 vol., Robert Laffont, Paris.

Furst Jill Leslie

1982 "Skeletonization in Mixtec Art: A Re-evaluation », in Elizabeth H. Boone (éd.), The Art and Iconography of Late Post-Classic central México, Dumbarton Oaks, Trustee for Harvard University, Washington, DC, pp. 207-225.

Furst Peter T.

1972 "El concepto huichol del alma ", in Peter T. Furst et Salomón Nahmad (éds), Mitos y arte huicholes, Sep/Setentas, Mexico, pp. 7-113.

GALinier Jacques

1990 La mitad del mundo. Cuerpo y cosmos en los rituales otomies, UNAM, IIA/CEMCA/INI, Mexico.

GaRCía Fray Gregorio

1981 Origen de los Indios de el Nuevo Mundo e Indias occidentales, FCE, Mexico [1607].

García de León Antonio

1969 «El universo de lo sobrenatural entre los nahuas de Pajapan, Veracruz », ECN, 8, pp. 279-311.

Gillow Eulogio G.

1889 Apuntes históricos, Imprenta del Sagrado Corazón de Jésus, Mexico.

GonZález Luis R.

1980 "La etnografia acaxee de Hernando de Santarén », Tlalocan, 8, pp. 355394. 
Graulich Michel

1987 Mythes et rituels du Mexique ancien préhispanique, Académie royale, Bruxelles.

1994 Montezuma ou l'apogée et la chute de l'empire aztèque, Fayard, Paris.

1997 "Chasse et sacrifice humain chez les Aztèques », Bulletin des séances de I'Académie royale des sciences d'Outre-mer, 43 (4), pp. 433-446.

1998 "La royauté sacrée chez les Aztèques de Mexico », ECN, 28, pp. 197-217.

2005 Le sacrifice humain chez les Aztèques, Fayard, Paris.

Grube Nikolai

2001 «Los nombres de los gobernantes mayas », Arqueología Mexicana, 9 (50), pp. $72-77$.

Gutiérrez del ÁNGel Arturo

2002 La peregrinación a Wirikuta: el gran rito de paso de los huicholes, INAH/Universidad de Guadalajara, Mexico.

HAMAYON Roberte

1998 "Le sens de l'“alliance" religieuse. "Mari" d'esprit, "femme" de dieu », Anthropologie et Société, Numéro spécial " Médiations chamaniques. Sexe et genre ", 22 (2), pp. 25-48.

2001a « Nier la mort, simuler l'amour et rappeler la vie ou le traitement funéraire du gibier abattu chez les peuples chasseurs de la forêt sibérienne ", in Liliane Bodson (éd.), La sépulture des animaux: concepts, usages et pratiques à travers le temps et l'espace. Contribution à l'étude de l'animalité, Colloque d'histoire des connaissances zoologiques, Université de Liège, Liège, pp. 107-128.

2001b «Tricks of the trade or how Siberian hunters play the game of lifeexchange ", in Caroline Gerschlager (éd.), Expanding the economic concept of exchange. Deception, self-deception and illusions, Kluwer Academic Publishers, Boston/Dordrecht/Londres, pp. 133-147.

Hémond Aline

1996 «Pratiques cynégétiques et nouveaux discours écologiques chez les Nahuas du Balsas (Mexique) ", JAIBA, revue d'Ethnobiologie, 38 (2), pp. 269-288.

HERNÁNDEZ Francisco

1959 Historia natural de Nueva España, in Obras completas, vol. 2 et 3, UNAM, Mexico.

HEYDEN Doris

1991 «La matriz de la tierra », in Johanna Broda, Stanislaw Iwaniszewski et Lucrecia Maupomé (éds), Arqueoastronomía y etnoastronomía en Mesoamérica, UNAM, IIH, Mexico, pp. 501-517.

HILL W. W.

1938 The agriculture and hunting methods of the Navaho Indians, Yale University Press, New Haven/Londres. 
Historia de los mexicanos por sus pinturas

1941 in Nueva colección de documentos para la historia de México, éd. Joaquín García Icazbalceta, Salvador Chavez Hayhoe, Mexico, pp. 209-240.

Historia tolteca-chichimeca

1976 éd. et trad. Paul Kirchhoff, Lina Odena Güemes et Luis Reyes García, INAH/SEP, Mexico.

Houston Stephen, Héctor Escobedo, Charles Golden, Andrew Scherer, Rosaura VÁzquez, Ana Lucía Arroyave, Fabiola QuiroA et Juan Carlos Meléndez

2006 « La Técnia and El Kinel : mounds and a monument upriver from Yaxchilan », Mexicon, 28 (5), pp. 87-93.

ICHON Alain

1969 La religion des Totonaques de la Sierra, CNRS, Paris.

JÄCKLEIN Klaus

1974 Un pueblo popoloca, trad. María Martínez Peñaloza, INI, Mexico.

JACKSON Robert H.

2002 " "Han ignorado la amorosa voz del Padre". Reconsiderando los orígenes del levantamiento de los chumash en 1824 en la California mexicana ", Desacatos, 10, pp. 77-93.

JANSEN Maarten

1989 "Nombres históricos e identidad étnica en los códices mixtecos », Revista Europea de Estudios Latinoamericanos y del Caribe, 47, pp. 65-87.

KanTOROWICZ Ernst

2000 Les deux corps du roi. Essai sur la théologie politique au Moyen Âge, in CEuves, Gallimard, Paris, pp. 643-1222 [1957].

LANDA Fray Diego de

1959 Relación de las cosas de Yucatán, éd. Ángel M. Garibay K., Porrúa, Mexico.

LAUNEY Michel

1980 Introduction à la langue et à la littérature aztèques, 2 vol., L'Harmattan, Paris.

LEÓN Alonso de

1961 Historia de Nuevo León, con noticias sobre Coahuila, Tamaulipas, Texas y Nuevo México, Universidad Autónoma de Nuevo León, Monterrey.

León-PortiLla Miguel

1959 «La historia del Tohueyo. Narración Erótica Náhuatl», ECN, 1, pp. 95-112.

LEOPOLD Starker A.

1959 Wildlife in Mexico, University of California Press, Berkeley/Los Angeles. 
Leyenda de los Soles

1992a in John Bierhorst, Codex Chimalpopoca. The Text in Nahuatl with a Glossary and Grammatical Notes, The University of Arizona Press, Tucson/Londres, pp. 85-100.

1992b in John Bierhorst, History and Mythology of the Aztecs. The Codex Chimalpopoca, The University of Arizona Press, Tucson/Londres, pp. 139-162.

Lienzos y Códice de Tepeticpac

1986 éd. Carmen Aguilera, Instituto Tlaxcaltera de la Cultura, Mexico.

Lizor Jacques

1976 Le cercle des feux. Faits et dits des Indiens yanomami, Seuil, Paris.

\section{López Austin Alfredo}

1994 Tamoanchan y Tlalocan, FCE, Mexico.

LóPEZ de Cogolludo Diego

1954-1955 Historia de Yucatán, 3 vol., Comisión de Historia, Campeche.

LóPEZ LuJÁN Leonardo

1993 Las ofrendas del Templo Mayor de Tenochtitlan, INAH, Mexico.

LOT-FALCK Eveline

$1953 \quad$ Les rites de chasse chez les peuples sibériens, Gallimard, Paris.

Lumholtz Carl

1986 El México desconocido, 2 vol., trad. Balbino Dávalos, INI, Mexico [1904].

McAnany Patricia A.

1995 Living with the ancestors. Kinship and kingship in Ancient Maya society, University of Texas Press, Austin.

MiLLeR Walter S.

1956 Cuentos mixes, INI, Mexico.

Molina Alonso de

1970 Vocabulario en lengua castellana y mexicana y mexicana y castellana, éd. Miguel León-Portilla, Porrúa, Mexico [1571].

Monod BeCquelin Aurore et Alain Breton

2002 La guerre rouge ou une politique maya du sacré. Un carnaval tzeltal au Chiapas, Mexique, CNRS Éditions, Paris.

Motolinía [ou Benavente] Fray Toribio

1971 Memoriales o Libro de las cosas de la Nueva España y de los naturales de ella, éd. Edmundo O'Gorman, UNAM, IIH, Mexico.

\section{Muñoz Camargo Diego}

1998 Historia de Tlaxcala. Ms. 210 de la Biblioteca Nacional de Paris, éd. Luis Reyes García avec la collaboration de Javier Lira Toledo, Gobierno del Estado de Tlaxcala, CIESAS/Universidad Autónoma de Tlaxcala, Mexico/Tlaxcala. 
MYERHOFF Barbara G.

1974 Peyote humt. The sacred journey of the Huichol Indians, Cornell University Press, Ithaca/Londres.

NAGY Christopher L. Von

1997 "Some comments on the Madrid deer-hunting almanacs », in Victoria R. Bricker et Gabrielle Vail (éds), Papers on the Madrid Codex, Tulane University, Middle American Research Institute, New Orleans, pp. 27-71.

NeFF Nuixa Françoise

2001 "La Lucerna y el Volcán Negro », in Johanna Broda, Stanislaw Iwaniszewski et Arturo Montero (éds), La montaña en el paisaje ritual, UNAM, IIH/INAH, Mexico, pp. 353-373.

NeURATH Johannes

2002 Las fiestas de la Casa Grande. Procesos rituales, cosmovisión y estructura social en una comunidad huichola, INAH/Universidad de Guadalajara, Mexico.

\section{Nowotny Karl Anton}

2005 Tlacuilolli. Style and contents of the Mexican pictorial mamuscripts with a catalog of the Borgia Group, éd. et trad. George A. Everett et Edward B. Sisson, University of Oklahoma Press, Norman [1961].

\section{OLIVIER Guilhem}

1997 Moqueries et métamorphoses d'un dieu aztèque : Tezcatlipoca, le « Seigneur au miroir fumant », Institut d'Ethnologie/CEMCA, Paris.

1999-2000 " Mixcoatl, "Serpent de Nuage", étude d'une divinité de l'ancien Mexique ", Annuaire de l'École Pratique des Hautes Études, 108, pp. 83-89.

2001 "Images et discours : à propos de quelques représentations de Mixcoatl, divinité des anciens Mexicains », in Pierre Ragon et Aline Hémond (éds), L'image au Mexique. Usages, appropriations et transgressions, L'Harmattan/ CEMCA, Paris, pp. 35-51.

2004 « De flechas, dardos y saetas. Mixcóatl y el simbolismo de las flechas en las fuentes nahuas ", in Ignacio Guzmán Betancourt, Pilar Máynez et Ascención Hernández de León-Portilla (éds), De historiografia lingüistica e historia de las lenguas, Siglo xxi/UNAM, IIF, Mexico, pp. 309-324.

2008 «Las tres muertes del nuevo tlatoani : una nueva interpretación de los ritos de entronización mexica », in Guilhem Olivier (éd.), Símbolos de poder en Mesoamérica, UNAM, IIH, IIA, Mexico, pp. 263-291.

Ortega Joseph de, S. J.

1996 «Libro I. Maravillosa reducción, y conquista de la Provincia de San Joseph del Gran Nayar, nuevo reino de Toledo ", in Francisco Javier Fluviá S. J. (éd.), Apostólicos afanes de la compañia de Jesús en su provincia de México, éd. Thomas Calvo et Jesús Jáuregui, édition en fac-similé, CEMCA/INI, Mexico, pp. 1-223 [1754]. 
PÉREZ de Ribas Andrés

$1992 \quad H i s t o r i a$ de los triumfos de nuestra santa fe entre gentes las más bárbaras y fieras del nuevo orbe, éd. Ignacio Guzmán Betancourt, Siglo xxI, Mexico [1645].

Pomar Juan Bautista de

1986 "Relación de Tezcoco ", in Relaciones geográficas del siglo xvI : México, vol. 3, éd. René Acuña, UNAM, IIA, Mexico, pp. 23-113.

Popol Vuh

1985 éd. et trad. Dennis Tedlock, Simon \& Schuster, New York.

Pozas Ricardo

1959 Chamula. Un pueblo indio de los Altos de Chiapas, Memorias del INI, vol. 8, Mexico.

Preuss Konrad Theodor

$1998 \quad F i e s t a$, literatura y magia en el Nayarit. Ensayos sobre coras, huicholes y mexicaneros, éd. et trad. Jesús Jáuregui et Johannes Neurath, INI/CEMCA, Mexico.

REDFIELD Robert et Alfonso Villa Rojas

1934 Chan Kom, a Maya village, Carnegie Institution of Washington, Pub. 448, Washington.

ReEnTs Budet Dorie

1994 Painting the Maya universe : royal ceramics of the Classic Period, Duke University Press, Durham/Londres.

Relación de Michoacán

1988 éd. Francisco Miranda, SEP, Mexico.

Relaciones geográficas del siglo xv: Guatemala

1982 éd. René Acuña, UNAM, IIA, Mexico.

Relaciones geográficas del siglo xvı : Antequera

19842 vol., éd. René Acuña, UNAM, IIA, Mexico.

Relaciones geográficas del siglo XV1 : México

1986 vol. 2, éd. René Acuña, UNAM, IIA, Mexico.

Relaciones geográficas del siglo xvI : Michoacán

1987 éd. René Acuña, UNAM, IIA, Mexico.

Relaciones geográficas del siglo xvı: Nueva Galicia

1988 éd. René Acuña, UNAM, IIA, Mexico.

Relaciones histórico-geográficas de la gobernación de Yucatán

19832 vol., éd. Mercedes de la Garza, UNAM, IIF, CEM, Mexico.

Reyes García Luis

1960 Pasión y muerte del Cristo Sol, Universidad Veracruzana, Xalapa. 


\section{Ruiz de Alarcón Hernando}

1987 " Tratado de las supersticiones y costumbres gentilicas que oy viuen entre los indios naturales desta Nueua España », in Fernando Benítez (éd.), El alma encantada, FCE, Mexico, pp. 125-223.

Ruz Mario Humberto

1996 «De lazos, flechas, trampas y cerbatanas. La caza en los vocabularios coloniales mayas », in Mayán Cervantes (éd.), Mesoamérica y Los Andes, CIESAS, Mexico, pp. 83-140.

SAHAGÚN Fray Bernardino de

1950-1981 Florentine Codex. General History of the things of New Spain, Fray Bernardino de Sahagún, éd. et trad. Charles E. Dibble et Arthur J. O. Anderson, The School of American Research and the University of Utah, Santa Fe, New Mexico.

1993 Primeros Memoriales, Facsimile edition, University of Oklahoma Press, Norman.

1997 Primeros Memoriales, éd. et trad. Thelma D. Sullivan, révision Henry B. Nicholson, Arthur J. O. Anderson, Charles E. Dibble, Eloise Quiñones Keber et Wayne Ruwet, University of Oklahoma Press, Norman.

Scholes France et Eleanor B. Adams

1938 Don Diego Quijada, alcalde mayor de Yucatán, 1561-1565, 2 vol., Antigua Librería Robredo de José Porrúa e Hijos, Mexico.

\section{SCHULTZE-JENA Leonhard}

1946 La vida y las creencias de los indigenas quiches de Guatemala, trad. Antonio Goubaud Carrera et Herbert D. Sapper, Anales de la Sociedad de Geografía e Historia de Guatemala, Guatemala.

1977 Mitos y leyendas de los pipiles de Izalco, trad. Gloria Menjivar Rieken et Armida Parada Fortin, Ed. Cuscatlán, San Salvador.

STARr Frederick

$1900 \quad$ Notes upon the ethnography of Southern Mexico, Proceedings of Davenport Academy of Natural Science, vol. VIII, Davenport, Iowa.

SugiYama Saburo K.

1991 «Descubrimientos de entierros y ofrendas dedicadas al Templo Viejo de Quetzalcóatl ", in Rubén Cabrera Castro, Ignacio Rodríguez García et Noel Morelos García (éds), Teotihuacan 1980-1982. Nuevas interpretaciones, INAH, Mexico, pp. 275-326.

SugiYama Saburo K. et Leonardo López LujÁN

2007 «Dedicatory burial/offering complexes at the Moon Pyramid, Teotihuacan. A preliminary report of 1998-2004 explorations ", Ancient Mesoamerica, 18, pp. 127-146.

SurRallés Alexandre

2003 Au caur du sens. Perception, affectivité, action chez les Candoshi, CNRS/Maison des Sciences de l'Homme, Paris. 
TAPIA Zenteno Carlos de

1985 Paradigma apologético y noticia de la lengua huasteca, éd. René Acuña, UNAM, IIF, Mexico.

TAube Karl Andreas

1988 "A study of Classic Maya scaffold sacrifice », in Elizabeth Benson et Gillett Griffin (éds), Maya iconography, Princeton University Press, Princeton, pp. 331-351.

The Selden Roll

1955 éd. Cottie A. Burland, Verlag. Gebr. Mann, Berlin.

THÉvet André

1905 «Histoyre du Mechique, manuscrit français inédit du Xvi ${ }^{\mathrm{e}}$ siècle », éd. E. de Jonghe, JSA, nouvelle série, 2, pp. 1-41.

Torquemada Fray Juan de

1975-1983 Monarquía Indiana, 7 vol., éd. Miguel León-Portilla, UNAM, IIH, Mexico.

TOWNSEND Richard F.

1987 "Coronation at Tenochtitlan » in Elizabeth H. Boone (éd.), The Aztec Templo Mayor, Dumbarton Oaks, Trustee for Harvard University, Washington, DC, pp. 371-411.

Tozzer Alfred M.

1982 Mayas y lacandones. Un estudio comparativo, INI, Mexico [1907].

VALERI Valerio

1994 "Wild victims : hunting as sacrifice and sacrifice as hunting in Huaulu ", History of Religions, 34-2, pp. 101-131.

VÁsQuEz Fray Francisco

1937-1944 Crónica de la provincia del Santísimo Nombre de Jesús de Guatemala de la Orden de Nuestro Seráfico Padre San Francisco, Biblioteca Goathemala, Guatemala, vol. 14-17.

Vázquez García Verónica et Ma. de Lourdes Godínez Guevara

2005 «Cambio social y estatus masculino en la cacería indigena. Un estudio de caso del sureste veracruzano ", Relaciones, 103, pp. 133-167.

Vernant Jean Pierre et Marcel Detienne (éds)

1979 La cuisine du sacrifice en pays grec, Gallimard, Paris.

Viveiros de CAstro Eduardo

1993 "Le marbre et le myrte. De l'inconstance de l'âme sauvage ", in Aurore Becquelin et Antoinette Molinié (éds), Mémoire de la tradition, Société d'Ethnologie, Nanterre, pp. 365-431.

Vogt Evon Z.

1979 Ofrendas para los dioses, trad. Stella Mastrangelo, FCE, Mexico. 


\section{WAGLEY Charles}

1957 Santiago Chimaltenango. Estudio antropológico-social de una commidad indigena de Huehuetenango, trad. Joaquin Noval, Seminario de Integración Social Guatemalteco, Guatemala.

\section{Williams García Roberto}

1972 Mitos tepehuas, SEP, Mexico.

WisDom Charles

1961 Los chortis de Guatemala, trad. Joaquin Noval, Seminario de Integración Social Guatemalteco, Guatemala.

\section{ZINGG Robert $M$.}

1982 Los Huicholes. Una tribu de artistas, 2 vol., trad. Celia Paschero, INI, Mexico. 